\title{
The role of unsteadiness in direct initiation of gaseous detonations
}

\author{
By CHRIS A. ECKETT, JAMES J. QUIRK† \\ AND JOSEPH E. SHEPHERD: \\ Graduate Aeronautical Laboratories, California Institute of Technology, Pasadena, \\ CA 91125, USA
}

(Received 5 January 1998 and in revised form 19 May 2000)

\begin{abstract}
An analytical model is presented for the direct initiation of gaseous detonations by a blast wave. For stable or weakly unstable mixtures, numerical simulations of the spherical direct initiation event and local analysis of the one-dimensional unsteady reaction zone structure identify a competition between heat release, wave front curvature and unsteadiness. The primary failure mechanism is found to be unsteadiness in the induction zone arising from the deceleration of the wave front. The quasi-steady assumption is thus shown to be incorrect for direct initiation. The numerical simulations also suggest a non-uniqueness of critical energy in some cases, and the model developed here is an attempt to explain the lower critical energy only. A critical shock decay rate is determined in terms of the other fundamental dynamic parameters of the detonation wave, and hence this model is referred to as the critical decay rate $(\mathrm{CDR})$ model. The local analysis is validated by integration of reactionzone structure equations with real gas kinetics and prescribed unsteadiness. The CDR model is then applied to the global initiation problem to produce an analytical equation for the critical energy. Unlike previous phenomenological models of the critical energy, this equation is not dependent on other experimentally determined parameters and for evaluation requires only an appropriate reaction mechanism for the given gas mixture. For different fuel-oxidizer mixtures, it is found to give agreement with experimental data to within an order of magnitude.
\end{abstract}

\section{Introduction}

When a large amount of energy is released in a small region of an unconfined combustible gas mixture, a strong spherical blast wave ensues from the initial point. As the blast expands and decays, two possible outcomes have been observed experimentally. First, the blast wave velocity may decay to an approximately constant value near the Chapman-Jouget (CJ) velocity of the mixture, in which case a self-supported spherical detonation has been successfully initiated in the gas. The other possibility is that the blast continues to decelerate below the CJ velocity and eventually decays away to an acoustic wave in the manner of a blast in a non-reacting gas. In this failed initiation event, the reaction zone decouples from the shock front and lags behind the shock, becoming a low-speed flame.

$\dagger$ Present address: Computing, Information, and Communications Division, Los Alamos National Laboratory, Los Alamos, NM 87545, USA.

$\ddagger$ Author to whom correspondence should be addressed. 
This method of detonation initiation by an overdriven shock wave has been termed direct initiation, as opposed to the other main form of initiation known as deflagration to detonation transition (DDT). The main variable believed to control the success or failure of direct initiation is the magnitude of the initial energy release, provided the energy deposition is sufficiently fast and the igniter sufficiently small. Experiments suggest that for a given combustible gas mixture at given uniform premixed initial conditions, the energy release must be above a certain level, known as the critical energy, to successfully initiate a detonation.

The same arguments apply for direct initiation of cylindrical detonations and planar detonations, except that the source is a line or plane respectively, and the critical energy is an energy per unit length or per unit area respectively. Most previous work has focused on the spherically symmetric direct initiation as this is the most fundamental geometry and the spherical critical energy is considered one of the best indicators of detonability or detonation sensitivity of combustible gas mixtures. It typically varies by several orders of magnitude between different mixtures, allowing simple ranking of mixture detonabilities without the need for highly accurate experiments.

Various attempts have been made to model the spherical critical energy in the past. An extensive review is given in Lee \& Higgins (1999). Zel'dovich, Kogarko \& Simonov (1956) were the first to present a theoretical discussion of the critical energy. They argued on the basis of the energy released inside a given spherical volume, the existence of a critical energy $E_{c}$, proportional to the cube of the reaction zone thickness. Although that paper did not give a satisfactory theory for the quantitative prediction of critical energies, it introduced the concept that the critical energy is a dynamic parameter of detonation and depends on the reaction kinetics of the combustible gas mixture.

Following Zel'dovich et al.'s findings, various workers produced phenomenological models that correlated the critical energy with other experimentally determined dynamic parameters of detonation, such as the cell width $\lambda$, the critical tube diameter $d_{c}$ and the hydrodynamic thickness $\Delta_{H}$. In all cases, the spherical critical energy was found to be proportional to the cube of the other dynamic parameter, consistent with Zel'dovich et al.'s theory. These models were reviewed by Lee $(1977,1984)$ and Benedick et al. (1986). This last work also compared the predictions of several models with experimental data for various fuel-air mixtures. These phenomenological models are based on experimental observations and the resulting equations merely correlate the critical energy to some other experimentally determined parameter. Admittedly, a parameter such as the cell width $\lambda$ is considerably easier to measure than the critical energy, so there is merit in such theories. However, it would be desirable to have a model that gives more insight into the underlying physical processes governing direct initiation, and provides an expression for the critical energy that can be evaluated without the need for experimental data.

The first attempt at such a rigorous theoretical model was made by $\mathrm{He} \&$ Clavin (1994). They assumed that the point-blast direct initiation problem could be adequately described by a quasi-steady analysis. The nonlinear curvature effect of the detonation front then provides the mechanism of failure. Excessive curvature prevents a sonic point from appearing at the rear of the reaction zone, and the decaying blast wave fails to evolve into the quasi-steady velocity-curvature relationship. We will refer to this model as the critical curvature model.

The dominant balance in He \& Clavin's model is competition between chemical heat release and front curvature. Such a quasi-steady model is popularly referred to as a $D_{n}-\kappa$ model (Stewart \& Bdzil 1988), where $D_{n}$ is the normal shock velocity and $\kappa$ is 
the local front curvature. These models are typically only applicable in a regime near the CJ velocity. The $D_{n}-\kappa$ concept was extended by Yao \& Stewart (1996) to a $\dot{D}_{n}-$ $D_{n}-\kappa$ model as well as a $\ddot{D}_{n}-\dot{D}_{n}-D_{n}-\kappa-\dot{\kappa}$ model, where the dots refer to differentiation with respect to time. These time-dependent models still assume that the dominant balance is between heat release and curvature, and that the detonation structure is characterized by a sonic point at the rear of the reaction zone. The unsteady terms are assumed to be small compared to the quasi-steady terms, restricting application of the models to slightly unsteady flow. There are a number of interesting consequences of these models, including cellular detonations (Stewart, Aslam \& Yao 1996).

In this paper we present an alternative model for direct initiation that arises from a detailed analysis of the unsteady reaction-zone structure. Our analytical approach is somewhat similar to Yao \& Stewart's in writing the governing equations with the unsteady terms as a perturbation on the steady flow. However, we make no assumptions regarding the size of different terms in the equations until they have been examined via numerical simulation results. As we shall demonstrate later in this paper, direct initiation cannot be described as slightly unsteady, and a more general treatment of the unsteady terms must be made, where they are not assumed to be a small perturbation. The unsteadiness of the decelerating leading shock wave is found to be the dominant mechanism causing failure in direct initiation.

The governing equations for flow along a particle path in the reaction zone are developed in $\S 2$. Numerical simulations of the spherical direct initiation problem with a one-step Arrhenius reaction rate law are outlined in $\S 3$, and used to examine the details of the flow in the reaction zone. Then in $\S 4$, analysis of the one-dimensional reaction-zone structure leads to the development of a local initiation model. A quasiunsteady computation of real-gas reaction zones is used to validate the local model in $\S 5$. In $\S 6$, the local initiation model is applied to the global initiation event to produce an analytical equation for the critical energy. Finally, this equation is compared with the critical curvature model and experimental data in $\S 7$.

\section{Reaction-zone structure equations}

Ignoring viscosity, heat transfer, diffusion and body forces, the governing equations are the reactive Euler equations. If the multi-dimensional nature of detonations is also ignored then a one-dimensional description is valid. In a fixed reference frame, the reactive Euler equations for flows with slab, cylindrical or spherical symmetry are given by

$$
\begin{aligned}
& \frac{\mathrm{D} \rho}{\mathrm{D} t}+\rho \frac{\partial u}{\partial r}+\frac{j}{r} \rho u=0, \quad \frac{\mathrm{D} u}{\mathrm{D} t}+\frac{1}{\rho} \frac{\partial P}{\partial r}=0, \\
& \frac{\mathrm{D} e}{\mathrm{D} t}-\frac{P}{\rho^{2}} \frac{\mathrm{D} \rho}{\mathrm{D} t}=0, \quad \frac{\mathrm{D} y_{k}}{\mathrm{D} t}=\Omega_{k},
\end{aligned}
$$

where $u, \rho, P$ and $e$ are the velocity, density, pressure and specific internal energy, $r$ is the distance from the coordinate origin, $t$ is the time, $j=0$ for planar flow, 1 for cylindrically symmetric flow and 2 for spherically symmetric flow, $y_{k}$ is the mass fraction of species $k$, and $\Omega_{k}$ is the production rate of species $k$, given by some kinetic rate law.

Using simple thermodynamic relations, the energy equation (2.1c) may be replaced by the adiabatic change equation from Fickett \& Davis (1979),

$$
\frac{\mathrm{D} P}{\mathrm{D} t}=c^{2} \frac{\mathrm{D} \rho}{\mathrm{D} t}+\rho c^{2} \dot{\sigma},
$$


where $c$ is the frozen sound speed, $\dot{\sigma}=\sum \sigma_{k} \Omega_{k}$ is the total thermicity with the sum over all species, and $\sigma_{k}$ is the thermicity coefficient of species $k$, given by

$$
\sigma_{k}=\left.\frac{1}{\rho c^{2}} \frac{\partial P}{\partial y_{k}}\right|_{e, \rho, y_{j \neq k}}=-\frac{1}{\rho c^{2}} \frac{\left.\left(\partial e / \partial y_{k}\right)\right|_{P, \rho, y_{j \neq k}}}{\left.(\partial e / \partial P)\right|_{\rho, y}} .
$$

The equations of motion can be rewritten in a reference frame attached to the shock using the following transformation:

$$
x=R(t)-r, \quad w(x, t)=U(t)-u(r, t),
$$

where $R$ and $U$ are the position and velocity of the shock in the fixed reference frame, and $w$ is the flow velocity in the shock-attached reference frame. For the remainder of this section, partial derivatives with respect to $t$ will indicate differentiation at constant $x$ as opposed to constant $r$. Then $(2.1 a, b),(2.2)$ and (2.1d) become

$$
\begin{aligned}
& \frac{\mathrm{D} \rho}{\mathrm{D} t}+\rho \frac{\partial w}{\partial x}+\frac{j}{R-x} \rho(U-w)=0, \quad \frac{\mathrm{D} w}{\mathrm{D} t}+\frac{1}{\rho} \frac{\partial P}{\partial x}=\frac{\mathrm{d} U}{\mathrm{~d} t}, \\
& \frac{\mathrm{D} P}{\mathrm{D} t}=c^{2} \frac{\mathrm{D} \rho}{\mathrm{D} t}+\rho c^{2} \dot{\sigma}, \quad \frac{\mathrm{D} y_{k}}{\mathrm{D} t}=\Omega_{k} .
\end{aligned}
$$

Equations $(2.4 a, b)$ can be written as

$$
\begin{aligned}
& \frac{\mathrm{D} \rho}{\mathrm{D} t}=-\frac{\rho}{w} \frac{\mathrm{D} w}{\mathrm{D} t}+\frac{\rho}{w} \frac{\partial w}{\partial t}-\frac{j}{R-x} \rho(U-w), \\
& \frac{\mathrm{D} P}{\mathrm{D} t}=-\rho w \frac{\mathrm{D} w}{\mathrm{D} t}+\frac{\partial P}{\partial t}+\rho w \frac{\mathrm{d} U}{\mathrm{~d} t} .
\end{aligned}
$$

Substituting (2.5) into $(2.4 c)$ gives

$$
\eta \frac{\mathrm{D} w}{\mathrm{D} t}=w \dot{\sigma}-\frac{j}{R-x} w(U-w)-M^{2} \frac{\mathrm{d} U}{\mathrm{~d} t}+\frac{\partial w}{\partial t}-\frac{w}{\rho c^{2}} \frac{\partial P}{\partial t},
$$

where the flow Mach number $M$ and sonic parameter $\eta$ are given by

$$
M=\frac{w}{c}, \quad \eta=1-M^{2} .
$$

Substituting (2.6a) into (2.5) gives

$$
\begin{aligned}
& \eta \frac{\mathrm{D} \rho}{\mathrm{D} t}=-\rho \dot{\sigma}+\frac{j}{R-x} \rho M^{2}(U-w)+\frac{\rho w}{c^{2}} \frac{\mathrm{d} U}{\mathrm{~d} t}-\frac{\rho w}{c^{2}} \frac{\partial w}{\partial t}+\frac{1}{c^{2}} \frac{\partial P}{\partial t}, \\
& \eta \frac{\mathrm{D} P}{\mathrm{D} t}=-\rho w^{2} \dot{\sigma}+\frac{j}{R-x} \rho w^{2}(U-w)+\rho w \frac{\mathrm{d} U}{\mathrm{~d} t}-\rho w \frac{\partial w}{\partial t}+\frac{\partial P}{\partial t} .
\end{aligned}
$$

Equations (2.6) are the solutions for the velocity, density and pressure gradients along a Lagrangian particle path behind the shock. We will refer to them as the reaction zone structure equations. In each equation, the first term on the right-hand side is the contribution from the chemical heat release, the second is that due to wave curvature, and the remaining terms represent the purely unsteady contribution. Retaining only the heat release term, the equations reduce to the Zel'dovich-Neumann-Doering (ZND) model of steady planar reacting flow (Fickett \& Davis 1979).

The simplest concept of detonation failure is a decoupling of the reaction zone from the shock front, or equivalently, the failure of particles to rapidly undergo reaction after they cross the shock. Since most reaction rate laws are strongly temperature 
dependent, the region of predominant reaction will be accompanied by a sharp temperature increase. Hence, the Lagrangian gradient of temperature will be of most interest when considering possible failure of the detonation. To compute the temperature gradient we need to invoke an equation of state.

Consider a system of ideal gases. The thermal equation of state is

$$
P=\rho R_{g} T,
$$

where $T$ is the temperature. $R_{g}$ is the mixture gas constant, given by

$$
R_{g}=\frac{\mathscr{R}}{W}=\mathscr{R} \sum \frac{y_{k}}{W_{k}},
$$

where $\mathscr{R}$ is the universal gas constant, $W$ is the mean molar mass of the mixture, and $W_{k}$ is the molar mass of species $k$. The frozen sound speed is

$$
c=\left(\frac{\gamma P}{\rho}\right)^{1 / 2},
$$

where $\gamma$ is the ratio of mixture specific heats. Equation (2.3) can be used to show that the thermicity coefficients are

$$
\sigma_{k}=\frac{1}{\gamma}\left(\frac{W}{W_{k}}-\frac{e_{k}}{C_{v} T}\right),
$$

where $e_{k}$ is the specific internal energy of species $k$, and $C_{v}$ is the mixture specific heat at constant volume. Taking the substantial derivative of $(2.7)$ and using $(2.6 b, c)$, (2.8) - (2.10) gives

$$
\begin{array}{r}
\eta C_{P} \frac{\mathrm{D} T}{\mathrm{D} t}=-\left(1-\gamma M^{2}\right) \sum e_{k} \Omega_{k}-\frac{c^{2}}{\gamma} \sum \frac{W}{W_{k}} \Omega_{k}+\frac{j}{R-x} w^{2}(U-w) \\
+w \frac{\mathrm{d} U}{\mathrm{~d} t}-w \frac{\partial w}{\partial t}+\frac{1}{\rho} \frac{\partial P}{\partial t},
\end{array}
$$

where $C_{P}$ is the mixture specific heat at constant pressure.

To enable analytical solution, we will now simplify the chemistry. Consider the one-step irreversible reaction, $\mathrm{A} \rightarrow \mathrm{B}$, where the upstream fluid is totally species $\mathrm{A}$, undiluted. The reactant and product are taken to be perfect gases (constant specific heat) and to have the same specific heats. So the specific internal energies of species $\mathrm{A}$ and $\mathrm{B}$ are

$$
e_{\mathrm{A}}=C_{v} T, \quad e_{\mathrm{B}}=C_{v} T-Q,
$$

where $Q$ is the heat of reaction. Define the progress variable $Z$ as the mass fraction of product $\mathrm{B}, Z=y_{\mathrm{B}}=1-y_{\mathrm{A}}$. Then, (2.11) becomes

$$
\eta C_{P} \frac{\mathrm{D} T}{\mathrm{D} t}=\left(1-\gamma M^{2}\right) Q \frac{\mathrm{D} Z}{\mathrm{D} t}+\frac{j}{R-x} w^{2}(U-w)+w \frac{\mathrm{d} U}{\mathrm{~d} t}-w \frac{\partial w}{\partial t}+\frac{1}{\rho} \frac{\partial P}{\partial t} .
$$

The kinetics are assumed to be governed by a first-order Arrhenius rate law with linear depletion,

$$
\frac{\mathrm{D} Z}{\mathrm{D} t}=k(1-Z) \exp \left(-\frac{E_{a}}{R_{g} T}\right),
$$

where $E_{a}$ is the activation energy per unit mass and $k$ is the pre-exponential rate 
multiplier. Then, (2.12) becomes

$$
\begin{aligned}
\left(1-M^{2}\right) C_{P} \frac{\mathrm{D} T}{\mathrm{D} t}=\left(1-\gamma M^{2}\right) Q k(1-Z) \exp ( & \left.-\frac{E_{a}}{R_{g} T}\right)+\frac{j}{R-x} w^{2}(U-w) \\
& +w \frac{\mathrm{d} U}{\mathrm{~d} t}-w \frac{\partial w}{\partial t}+\frac{1}{\rho} \frac{\partial P}{\partial t} .
\end{aligned}
$$

Equation (2.13) is the temperature-reaction-zone structure equation for the one-step reaction model, and has the form of an energy equation. As before, the first term on the right-hand side is the contribution from heat release, the second term is that from wave curvature and the remaining terms are due to unsteadiness. We should emphasize that in this equation and the earlier reaction zone structure equations (2.6), the left-hand side contains a Lagrangian derivative. This could be divided into time and space partial derivatives, and one could argue that the term arising from the partial time derivative should be moved to the right-hand side and grouped with the other unsteady derivatives. This would ensure that all unsteadiness appears in the group of unsteady terms on the right-hand side and would permit direct comparison with the quasi-steady equations. In fact, such a comparison has been made and is discussed in the following section. However, for the purpose of analysis, we choose to write the equations in the Lagrangian reference frame, where the unsteady terms on the right-hand side are only the unsteadiness that a particle sees, not the unsteadiness we traditionally think of in an Eulerian reference frame. The Lagrangian reference frame is a more natural choice when considering the reaction in a detonation as a convected adiabatic chain-thermal explosion. For the remainder of the paper, we shall use the terminology 'unsteady terms' or 'unsteadiness' to denote only the unsteady terms on the right-hand side of the reaction-zone structure equations (2.6) and (2.13). Note that the unsteady terms in (2.13) are proportional to the unsteady terms in the dilatational rate equation, $(2.6 b)$, so they may be interpreted as arising from the dilatational rate in the absence of heat release and curvature.

For a decelerating wave such as the blast wave in a direct initiation event, the unsteadiness expression in the energy equation (2.13) is of opposite sign to the heat release term. Thus the reaction may quench if the wave is decelerating too rapidly. For a convex-upstream wavefront such as the blast wave in a cylindrical or spherical direct initiation, the steady curvature term in (2.13) is of the same sign as the heat release term and so cannot possibly quench the reaction without the additional presence of unsteadiness. Note that a cylindrical or spherical blast wave flow will always be unsteady, even if the blast is propagating at constant velocity, since its curvature is changing with time. The time dependence of curvature can be an important effect, but it is important to realize that it appears in the unsteadiness expression in (2.13), not in the curvature term. For a slab symmetry direct initiation, there is no curvature term at all so, again, curvature cannot quench the reaction. Note that the opposite trends occur in the velocity, density and pressure reaction-zone structure equations (2.6), namely for the direct initiation problem the unsteadiness is of the same sign as the heat release while the curvature is of opposite sign. However the strong nonlinear temperature dependence of the reaction rate makes temperature the critical variable. The relative sizes and behaviour of the terms in (2.13) will be examined directly via numerical simulations in the next section. The goal is to identify the dominant balance in the direct initiation problem and any simplifying assumptions regarding the behaviour of the terms in (2.13) that would permit further analytical work. 


\section{Numerical simulations}

\subsection{Computational details}

Numerical simulations of the spherical blast wave initiation problem have been computed, using the one-step irreversible reaction described in $\S 2$. For this reaction model, the reactive Euler equations for flows with spherical symmetry, in a fixed reference frame and in non-dimensional conservative form, are

$$
\frac{\partial \boldsymbol{W}}{\partial \tilde{t}}+\frac{\partial \boldsymbol{F}}{\partial \tilde{r}}=\boldsymbol{G}+\boldsymbol{S},
$$

where

$$
\begin{gathered}
\boldsymbol{W}=\left(\begin{array}{c}
\tilde{\rho} \\
\tilde{\rho} \tilde{u} \\
\tilde{E}_{t} \\
\tilde{\rho} Z
\end{array}\right), \boldsymbol{F}=\left(\begin{array}{c}
\tilde{\rho} \tilde{u} \\
\tilde{\rho} \tilde{u}^{2}+\tilde{P} \\
(\tilde{E} t+\tilde{P}) \tilde{u} \\
\tilde{\rho} \tilde{u} Z
\end{array}\right), \boldsymbol{G}=-\frac{2}{\tilde{r}}\left(\begin{array}{c}
\tilde{\rho} \tilde{u} \\
\tilde{\rho} \tilde{u}^{2} \\
\left(\tilde{E}_{t}+\tilde{P}\right) \tilde{u} \\
\tilde{\rho} \tilde{u} Z
\end{array}\right), \\
\boldsymbol{S}=\left(\begin{array}{c}
0 \\
0 \\
0 \\
\tilde{k} \tilde{\rho}(1-Z) \mathrm{e}^{-\tilde{E}_{a} / \tilde{T}}
\end{array}\right) .
\end{gathered}
$$

$\boldsymbol{W}$ is the conservative solution vector, $\boldsymbol{F}$ is the convective flux, $\boldsymbol{G}$ and $\boldsymbol{S}$ are the geometry and reaction source terms respectively, and $E_{t}=\rho\left(e+u^{2} / 2\right)$ is the total energy per unit volume. The dimensional flow variables have been made non-dimensional as follows:

$$
\begin{gathered}
u_{r e f} \equiv\left(R_{g} T_{0}\right)^{1 / 2}, \quad \tilde{u} \equiv \frac{u}{u_{r e f}}, \quad \tilde{\rho} \equiv \frac{\rho}{\rho_{0}}, \quad \tilde{P} \equiv \frac{P}{P_{0}}, \\
\tilde{T} \equiv \frac{T}{T_{0}}, \quad \tilde{e} \equiv \frac{e}{R_{g} T_{0}}, \quad \tilde{E}_{t} \equiv \frac{E_{t}}{P_{0}}, \quad \tilde{E}_{a} \equiv \frac{E_{a}}{R_{g} T_{0}},
\end{gathered}
$$

where subscript 0 denotes the uniform conditions upstream of the shock. In the numerical simulations, $\tilde{k}$ is an arbitrary parameter that merely defines the spatial and temporal scales. It has been chosen such that for a planar CJ wave, the half-reaction length $\Delta_{1 / 2}$ is scaled to unit length, that is,

$$
\tilde{r} \equiv \frac{r}{\Delta_{1 / 2}}, \quad t_{r e f} \equiv \frac{\Delta_{1 / 2}}{u_{r e f}}, \quad \tilde{t} \equiv \frac{t}{t_{r e f}}, \quad \tilde{k} \equiv k t_{r e f} .
$$

The non-dimensional equations of state are

$$
\tilde{P}=\tilde{\rho} \tilde{T}, \quad \tilde{e}=\frac{1}{\gamma-1} \tilde{T}-Z \tilde{Q},
$$

where $\tilde{Q} \equiv Q / R_{g} T_{0}$.

The numerical integration was performed using operator splitting, with the algorithm

$$
\boldsymbol{W}^{n+1}=\mathscr{L}_{S} \mathscr{L}_{F G} \boldsymbol{W}^{n},
$$

where the superscript indicates the number of timesteps. When integrated in a uniform grid with a cell-centred, finite difference formulation, the convective and geometry source operator $\mathscr{L}_{F G}$ can be written as

$$
\boldsymbol{W}_{i}^{n+1}=\boldsymbol{W}_{i}^{n}-\frac{\Delta \tilde{t}}{\Delta \tilde{r}}\left(\boldsymbol{F}_{i+1 / 2}^{n}-\boldsymbol{F}_{i-1 / 2}^{n}\right)+\Delta \tilde{t} \boldsymbol{G}_{i}^{n},
$$


where $\Delta \tilde{t}$ is the timestep and $\Delta \tilde{r}$ is the cell size. The subscript indicates the spatial cell number. $\boldsymbol{F}_{i+1 / 2}^{n}$ is the flux at the interface between cells $i$ and $i+1$, and should be some conservative upwinding flux. In this work, we employed Roe's approximate Riemann solver (Roe 1986) for the convective flux, using Glaister's (1988) implementation for a general equation of state. Second-order temporal and spatial accuracy was obtained via min-mod flux limiting, and the scheme was made entropy-satisfying with Harten's entropy fix. The integration of the geometry term was only first-order time accurate. The benefit in making this second-order would be minimal given the small effect of these terms (see $\$ 3.2$ ).

Finally, the reaction source operator $\mathscr{L}_{S}$ involves the integration of the equation,

$$
\frac{\mathrm{d} \boldsymbol{W}}{\mathrm{d} \tilde{t}}=\boldsymbol{S},
$$

which reduces to

$$
\frac{\mathrm{d} Z}{\mathrm{~d} \tilde{t}}=\tilde{k}(1-Z) \mathrm{e}^{-\tilde{E}_{a} / \tilde{T}},
$$

with $\tilde{\rho}, \tilde{u}$ and $\tilde{e}$ constant. If the temperature was constant for this step, (3.2) could be integrated exactly. In this work, we performed the integration using a nominally second-order time accurate predictor-corrector scheme. Equation (3.2) was first integrated for a half-timestep, with the temperature held constant. This gave an estimate for the average mass fraction in the timestep, $Z^{n+1 / 2}$. The temperature $\tilde{T}^{n+1 / 2}$ was then computed from the caloric equation of state $(3.1 b)$, noting that $\tilde{e}$ is fixed for this step. Finally, (3.2) was integrated for the whole timestep, using the average temperature $\tilde{T}^{n+1 / 2}$.

The flow solver was incorporated into the Amrita CFD programming system (Quirk 1998), making use of Amrita's adaptive mesh refinement (AMR) algorithm. The simulations presented in this paper used four levels of grid refinement, with refinement ratios of 4 in each case. Refinement was performed around the shock, where the pressure gradient exceeded a specified threshold, and in the reaction zone, where the species gradient exceeded another threshold. The refinement criteria were chosen to produce a finely resolved shock and a reaction zone with at least 50 mesh cells per half-reaction length.

The choice of computational cases to study was made on the basis of the following argument. Throughout the analysis in this paper, it is implicitly assumed that the detonation wave is hydrodynamically stable. Previous computations by He (1996) on spherical detonation initiation with Arrhenius reaction rate demonstrated that instability provides a secondary means of detonation quenching. To isolate the purely gasdynamic quenching mechanism, we chose to perform computations only with stable or slightly unstable mixtures. When slightly unstable, the instability growth rate is sufficiently slow that the gasdynamic quenching still dominates in the short times involved. Using the normal mode stability analysis method of Lee \& Stewart (1990), the neutral stability curves for one-dimensional planar CJ detonations have been computed for various ratios of specific heat and are plotted in figure 1. Throughout this paper, the subscript $C J$ will be used to denote flow variables for a detonation travelling at CJ velocity, so $M_{0_{C J}}$ denotes the free-stream Mach number $M_{0}$ for a CJ wave. $\theta$ is the activation energy normalized by the post-shock temperature $T_{s}$,

$$
\theta \equiv \frac{E_{a}}{R_{g} T_{s}}
$$

so $\theta_{C J}$ denotes the value of $\theta$ for a wave travelling at $C J$ velocity. When plotted in the 


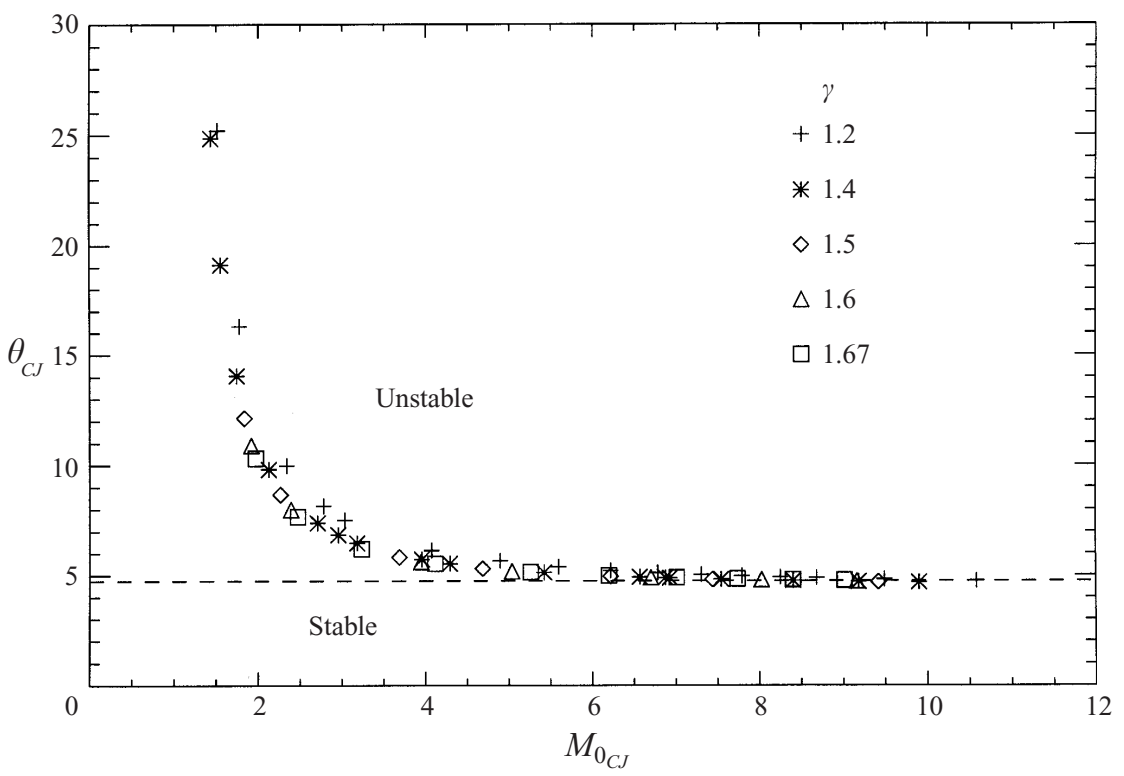

FIGURE 1. Neutral stability curve for planar CJ detonations with one-step Arrhenius rate law.

$\tilde{Q}-\tilde{E}_{a}$ plane as originally done by Lee \& Stewart (1990), the neutral stability curves for each value of $\gamma$ are different, but when plotted in the $\theta_{C J}-M_{0_{C J}}$ plane as in figure 1 , they essentially collapse to a single curve. Furthermore, for strong detonations with large values of $M_{0_{C J}}$, the neutral stability curve asymptotes to a constant value of $\theta_{C J} \approx 4.74$. In this regime, the stability of the wave is then a function of $\theta_{C J}$ only, an example of the dominant effect of $\theta$ for the Arrhenius reaction rate model. A further effect of $\theta$ is in the shape of the ZND reaction zone profile; the larger $\theta$, the more the ZND profile approaches that of a square-wave, with a near-constant state induction zone followed by a rapid energy release. This type of reaction-zone structure is typical of that observed in computations of real hydrocarbon mixtures. Hence it would be desirable to use a mixture with such a ZND profile in these computations. The need to maximize $\theta$ for a suitable ZND profile yet still remain stable or near stable resulted in the choice to examine near-critically stable mixtures. With this restriction and figure 1 in mind, the range of behaviour for the Arrhenius reaction rate model can be represented by just a single choice of $M_{0_{C J}}$ and $\theta_{C J}$. However, ZND calculations also indicate that for the same value of $M_{0_{C J}}$ and $\theta_{C J}$, lower values of $\gamma$ produce reaction zone profiles slightly closer to a square-wave. The dependence upon $\gamma$ is weak, but cannot be ignored given the desire to achieve a square-wave-like profile.

Considering the arguments presented in the previous paragraph, two parameter sets were chosen for computational investigation. They are listed in table 1 . The subscript $v N$ denotes the post-shock state (von Neumann conditions) for a wave travelling at CJ velocity, while the subscript $C J$ on the state variables $\tilde{P}$ and $\tilde{T}$ denotes the equilibrium state at the rear of the reaction zone for a wave travelling at CJ velocity. The two cases have the same value of $M_{0_{C J}}$ and close to the same value of $\theta$, but have different values of $\gamma$. Both cases are marginally unstable.

At early times in the flow, the blast wave will be a very strong shock, and the chemical energy released into the flow will be negligible compared to the blast source energy. Therefore, the flow will be closely approximated by the similarity solution for 


$\begin{array}{clcc} & \text { Case } & \mathrm{A} & \mathrm{B} \\ \text { Independent } & \gamma & 1.2 & 1.4 \\ \text { quantities } & \tilde{Q} & 22.5 & 12 \\ & \tilde{E}_{a} & 17 & 25 \\ \text { Dependent } & \tilde{k} & 70.8 & 108.3 \\ \text { quantities } & \tilde{U}_{C J} & 4.70 & 5.08 \\ & M_{0 J} & 4.29 & 4.29 \\ & \theta_{C J} & 6.05 & 5.54 \\ & \tilde{P}_{v N} & 20.0 & 21.3 \\ & \tilde{P}_{C J} & 10.5 & 11.2 \\ & \tilde{T}_{v N} & 2.81 & 4.52 \\ & \tilde{T}_{C J} & 6.00 & 6.76\end{array}$

TABLE 1. Input fluid and chemical parameters for the numerical simulations.

a non-reacting strong point blast with zero back-pressure (Taylor 1950; Sedov 1959). For a constant- $\gamma$ perfect gas, this point-blast theory (PBT) similarity solution is given by

$$
\begin{gathered}
R=\left(\frac{E_{\text {source }}}{A_{2} \rho_{0}}\right)^{1 / 5} t^{2 / 5}, \quad U=\frac{\mathrm{d} R}{\mathrm{~d} t}=\frac{2}{5}\left(\frac{E_{\text {source }}}{A_{2} \rho_{0}}\right)^{1 / 2} R^{-3 / 2}, \\
u_{s}=\frac{2}{\gamma+1} U, \quad \rho_{s}=\frac{\gamma+1}{\gamma-1} \rho_{0}, \quad P_{s}=\frac{2}{\gamma+1} \rho_{0} U^{2}, \\
\frac{u}{u_{s}}=f\left(\frac{r}{R}\right), \quad \frac{\rho}{\rho_{s}}=g\left(\frac{r}{R}\right), \quad \frac{P}{P_{s}}=h\left(\frac{r}{R}\right),
\end{gathered}
$$

where subscript $s$ denotes conditions immediately after the shock, $E_{\text {source }}$ is the initial energy release, and $A_{2}$ is the energy integral constant, which is a function of $\gamma$. Korobeinikov (1991) lists the functions $f(r / R), g(r / R)$ and $h(r / R)$, as well as a correlation for $A_{2}$, accurate to $0.31 \%$ in the range $1.2 \leqslant \gamma \leqslant 2.0$,

$$
A_{2}=0.31246(\gamma-1)^{-1.1409-0.11735 \log _{10}(\gamma-1)} .
$$

The initial condition used in the numerical simulations was the PBT similarity solution, applied at an initial shock radius $R_{\text {source }}$ much less than the shock radius of the critical flow regime later in the simulation. Numerical difficulties associated with the strong shock wave and the singularity at the origin in the PBT similarity solution placed a lower bound on the choice of the initial shock radius. However, in all computational cases presented here, the chemical energy inside the initial source region,

$$
E_{\text {chem }}=\frac{4}{3} \pi R_{\text {source }}^{3} \rho_{0} Q,
$$

was less than $3 \%$ of the source energy $E_{\text {source }}$, so the application of the non-reacting PBT was valid.

Consistent with the earlier normalizations, the non-dimensional source energy $\tilde{E}_{\text {source }}$ is defined by

$$
\tilde{E}_{\text {source }} \equiv \frac{E_{\text {source }}}{P_{0} \Delta_{1 / 2}^{3}}
$$



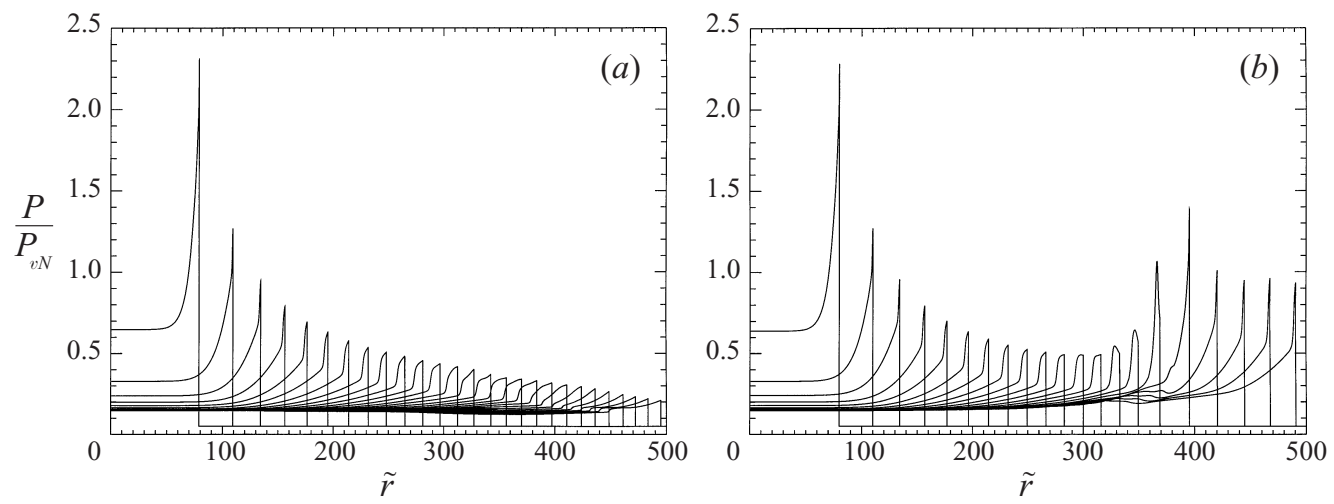

FIGURE 2. Spatial pressure profiles for case A, at roughly equal timesteps. (a) $\tilde{E}_{\text {source }}=166 \times 10^{6}$; (b) $\tilde{E}_{\text {source }}=169 \times 10^{6}$.

\subsection{Computational results}

Spatial pressure profiles are plotted in figure 2 for case A from table 1, with two different source energies. The first computation, with $\tilde{E}_{\text {source }}=166 \times 10^{6}$, fails to initiate, so this represents a subcritical initiation energy. As the wave decays to the CJ state, where $P_{s}=P_{v N}$, the von Neumann spike immediately behind the shock decreases in size and the reaction zone lengthens. The post-shock pressure continues to decay to well below the von Neumann pressure, and the von Neumann spike disappears, signifying failure to initiate a detonation. In figure $2(b)$, where $\tilde{E}_{\text {source }}=169 \times 10^{6}$, the early profiles closely match those in figure $2(a)$. But at around $\tilde{R}=300$, the post-shock pressure begins to rise, overshooting $P_{v N}$, before settling back down to around $P_{v N}$. It then remains close to steady, indicating a spherical detonation has been successfully initiated. Hence this source energy is a supercritical initiation energy. The mechanism causing the re-initiation explosion in figure $2(b)$ appears to be the formation and amplification of a pressure pulse at the rear of the reaction zone. This mechanism has also been observed in previous numerical simulations (Clarke, Kassoy \& Riley 1986; Clarke et al. 1990; Mazaheri 1997).

The location and velocity of the leading shock were determined as follows. The shock pressure $\tilde{P}_{s}$ was first evaluated approximately as the instantaneous local maximum in the pressure profile just behind the shock. This local maximum exists because the pressure behind the shock decreases as a result of the exothermic reaction and/or geometric expansion. The shock location $\tilde{R}$ was then determined as the interpolated position in the spatial pressure profile for which the pressure was $\left(\tilde{P}_{0}+\tilde{P}_{s}\right) / 2$, roughly the midpoint of the numerically smeared shock. Having computed this at many timesteps in the computation, the shock velocity was finally determined in a postprocessing operation by a second-order differentiation of the data points $\tilde{R}(\tilde{t})$.

Figure 3 shows the velocity of the leading shock plotted against the shock radius, for case A with several different source energies. In the successfully initiated cases, the mild instability of the detonation wave is evident. However, the instability takes a sufficiently long time to develop that it only appears after the detonation has successfully initiated. Hence, the instability does not seem to influence the gasdynamic initiation process significantly. The two near-critical curves, $\tilde{E}_{\text {source }}=166 \times 10^{6}$ and $169 \times 10^{6}$, begin to deviate significantly at about $\tilde{R}=225$, where $U / U_{C J}=0.75$. This point is the critical point of interest for these near-critical initiations, for it is here 


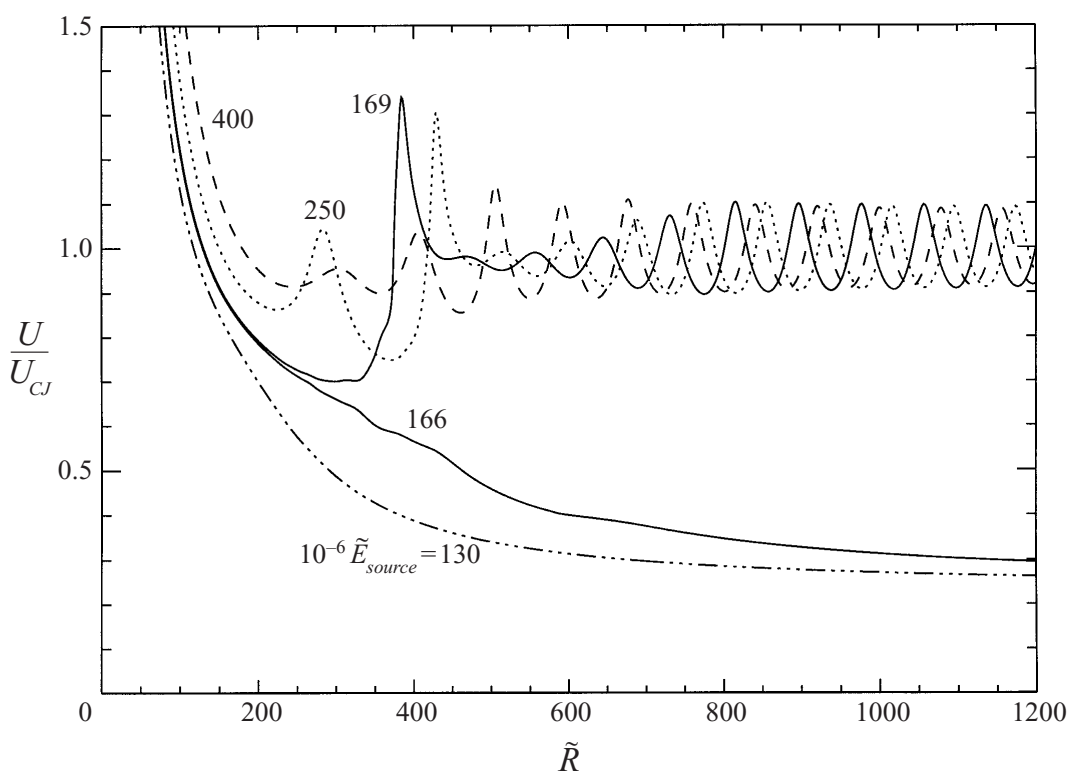

FIGURE 3. Leading shock velocity versus position for case A, with several different source energies. $\tilde{E}_{\text {source }}=130 \times 10^{6}, 166 \times 10^{6}, 169 \times 10^{6}, 250 \times 10^{6}, 400 \times 10^{6}$.

that failure or success is determined in the detonation initiation process. Everything after this, including the re-initiation mechanism for the supercritical case, is irrelevant if we are only concerned with the critical energy. This is in contrast to the proposition of Lee \& Higgins (1999) that any model of initiation criteria must address the mechanism of reacceleration of the decaying shock and transition to detonation. Our simulations indicate that it is possible to form an estimate of the critical initiation energy by examining the simpler problem of the failure mechanism involved in decoupling the reaction zone from the decaying blast wave. It is interesting to note that the critical point occurs well before the formation of the pressure pulse in figure 2(b). This suggests that the pressure pulse and the associated 'quasi-steady' (Lee \& Higgins 1999) portion of the velocity profile are not the underlying factors controlling initiation determination, but are merely the mechanisms by which successful initiation proceeds.

The position of the leading shock, the loci of $5 \%$ and $95 \%$ reaction, and the sonic surface are plotted against time in figure 4, for the two near-critical cases of the previous figure. In figure $4(a)$, the reaction zone is initially closely coupled to the shock wave when the shock is very strong, but it later detaches, indicating that the detonation has failed and the reaction has quenched. By contrast, the reaction zone remains closely coupled to the shock wave in figure $4(b)$, indicating the successful initiation of a quasi-steady detonation.

The sonic surfaces in figure 4 have been defined as the loci of points for which the flow is sonic with respect to the shock front at a given instant in time. The physically significant sonic point occurs when the flow is sonic with respect to the rear of the reaction zone, the limiting condition for which small disturbances can propagate into the reaction zone from the trailing expansion wave. However there is no simple way to determine the location of the rear of the reaction zone. In steady flow, the rear 

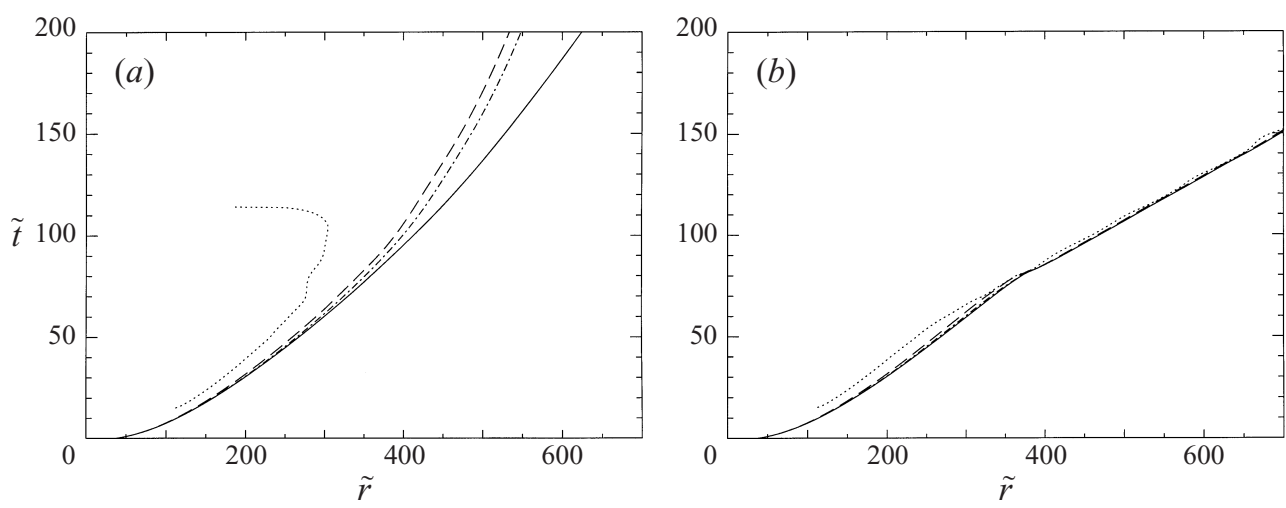

FIGURE 4. Leading shock position, reaction loci and sonic point location versus time, for case A: - , shock; $-\cdot-\cdot, 5 \%$ reaction;,$--- 95 \%$ reaction; $\cdots \cdots$, sonic point. (a) $\tilde{E}_{\text {source }}=166 \times 10^{6}$; (b) $\tilde{E}_{\text {source }}=169 \times 10^{6}$

of the reaction zone travels at the same velocity as the shock front, and these two sonic point definitions are equivalent, but they may differ in unsteady flow. For this reason, the sonic surface plotted in the $r-t$ diagrams cannot be regarded as the critical factor determining detonation initiation or failure. Its relevance is simply that it must eventually appear at the rear of the reaction zone if a quasi-steady, quasi-planar detonation is formed.

The reaction-zone structure equations in $\S 2$ described the evolution of quantities along particle paths. To examine the behaviour of these equations in the numerical simulations of the direct initiation problem, it was necessary to extract Lagrangian particle path data from the Eulerian flow solution. This was done by specifying some initial particle locations and then, in a non-intrusive fractional step of the flow integration, interpolating the particles' paths through the $r-t$ solution field, using the local flow velocity. The particle positions and flow variables at those locations were then output as functions of time.

For case A with $\tilde{E}_{\text {source }}=160 \times 10^{6}$, a slightly subcritical energy, figure 5 shows the paths of ten sample particles that cross the leading shock around the time of detonation failure. The plot also shows the shock and partial reaction region, as in the previous $r-t$ diagrams of figure 4 . The earlier particles traverse the reaction zone rapidly, indicating that the flow is still detonating at this stage. By about particle 6, the reaction time has grown significantly, suggesting that the wave is failing here. The last particles never reach the reaction zone in the time plotted. Note that the partial reaction lines are essentially parallel to the streamlines at the late times, indicating that the reaction has completely quenched by then.

Figure 6 shows the temperature as a function of time along the same ten particle paths. The first few reach thermal runaway quickly, but by the sixth or seventh particle path, the explosion time has grown significantly. The last particles merely cool gradually and never react. The slight negative temperature gradient along the later particle paths immediately after the shock is the forcing of the unsteadiness, as discussed in $\S 2$. It is this gradient that prevents the particles from undergoing thermal runaway.

The magnitude of the various competing terms in the temperature reaction zone structure equation was next examined in the numerical simulations. In the non- 


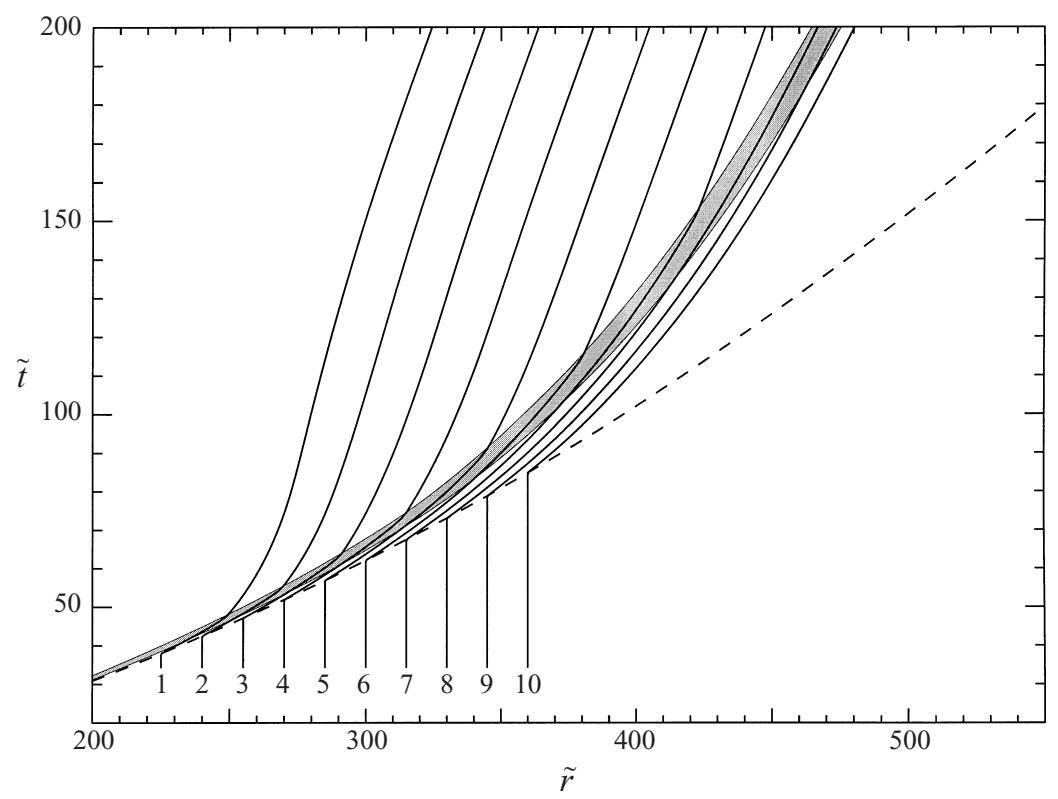

FIGURE 5. Particle paths for ten sample particles in case A, with $\tilde{E}_{\text {source }}=160 \times 10^{6}$. Shock (dashed line); $5 \%$ to $95 \%$ reaction (shaded region); particle paths (solid lines).

dimensional notation of $\S 3.1$, the temperature equation (2.13) for spherical flow $(j=2)$ becomes

$$
\begin{aligned}
\underbrace{\left(1-M^{2}\right) \frac{\gamma}{\gamma-1} \frac{\mathrm{D} \tilde{T}}{\mathrm{D} \tilde{t}}}_{\text {total }}= & \underbrace{\left(1-\gamma M^{2}\right) \tilde{Q} \tilde{k}(1-Z) \exp \left(-\frac{\tilde{E}_{a}}{\tilde{T}}\right)}_{\text {heat release }}+\underbrace{\frac{2}{\tilde{r}} \tilde{w}^{2}(\tilde{U}-\tilde{w})}_{\text {curvature }} \\
& +\underbrace{\tilde{w} \frac{\mathrm{d} \tilde{U}}{\mathrm{~d} \tilde{t}}-\tilde{w} \frac{\partial \tilde{w}}{\partial \tilde{t}}+\frac{1}{\tilde{\rho}} \frac{\partial \tilde{P}}{\partial \tilde{t}}}_{\text {unsteadiness }} .
\end{aligned}
$$

The unsteadiness terms were evaluated as the residual of this equation. As a consistency check, they were also evaluated with the aid of the mass and momentum conservation equations (2.5), in appropriate non-dimensional form, which gives

$$
\tilde{w} \frac{\mathrm{d} \tilde{U}}{\mathrm{~d} \tilde{t}}-\tilde{w} \frac{\partial \tilde{w}}{\partial \tilde{t}}+\frac{1}{\tilde{\rho}} \frac{\partial \tilde{P}}{\partial \tilde{t}}=\frac{1}{\tilde{\rho}}\left(\frac{\mathrm{D} \tilde{P}}{\mathrm{D} \tilde{t}}-\tilde{w}^{2} \frac{\mathrm{D} \tilde{\rho}}{\mathrm{D} \tilde{t}}\right)-\frac{2}{\tilde{r}} \tilde{w}^{2}(\tilde{U}-\tilde{w}) .
$$

The right-hand side of this equation was evaluated directly from the Lagrangian particle path data. The Lagrangian derivatives $\mathrm{D} \tilde{T} / \mathrm{D} \tilde{t}, \mathrm{D} \tilde{P} / \mathrm{D} \tilde{t}$ and $\mathrm{D} \tilde{\rho} / \mathrm{D} \tilde{t}$ in (3.5) and (3.6) were evaluated in a postprocessing operation by a second-order differentiation of the particle path data points $\tilde{T}(\tilde{t}), \tilde{P}(\tilde{t})$ and $\tilde{\rho}(\tilde{t})$.

The terms in (3.5) have been computed along the same ten particle paths as in figure 5, and are plotted in figure 7 for a selection of the particles. The left-hand border of each plot is the instant in time when the particle crosses the shock. For the particles prior to or at failure (particles 1,5 and 6), it is clear that the curvature term makes a negligible contribution to the temperature gradient when compared with the magnitudes of the other terms on the right-hand side of (3.5). By contrast, 


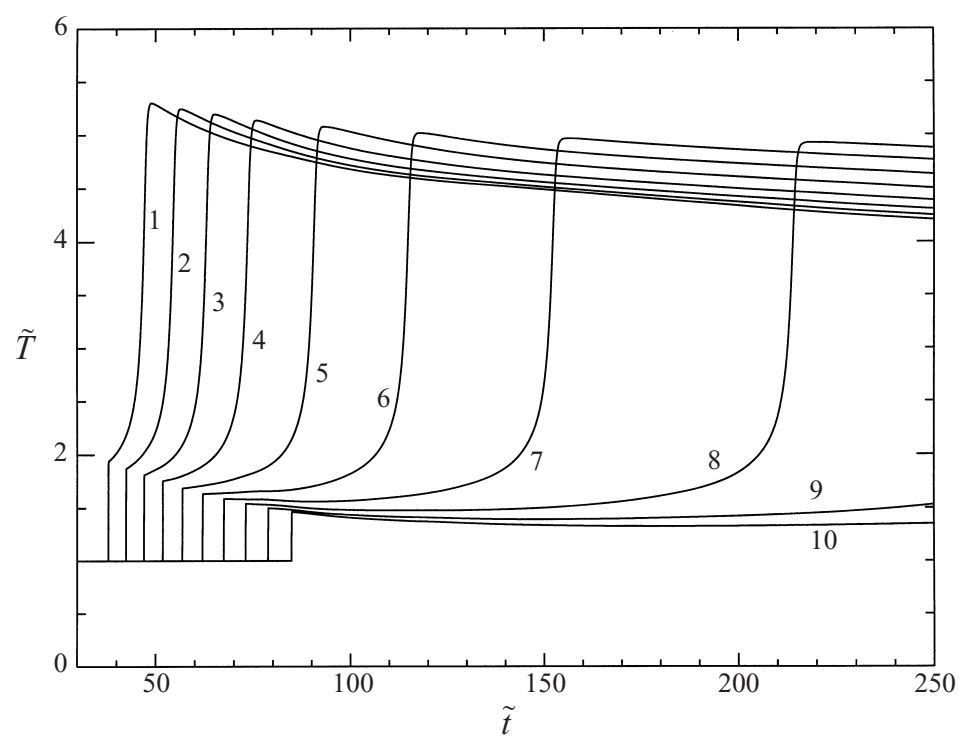

FIgURE 6. Temperature histories along the same ten particle paths as in figure 5, for case A with $\widetilde{E}_{\text {source }}=160 \times 10^{6}$.

the contribution from unsteadiness is significant. Along particle paths 1 and 5 , the unsteadiness is a negative forcing that reduces the total temperature gradient below that due to heat release, although it is not strong enough to prevent reaction. For particle path 6 , the unsteadiness is initially about equal to the heat release, causing the total gradient to be almost zero, and the reaction nearly quenches. By particle path 10, the unsteadiness dominates the heat release, and the reaction is completely quenched. A final observation is that the unsteadiness expression is almost constant along each particle path, within the induction zone. This is true for all particles before detonation failure, that is, all in figure 7 except particle path 10 . These important observations regarding the contributions of curvature and unsteadiness to the Lagrangian temperature derivative will be used to develop a local initiation model in the following section.

In $\S 2$, it was noted that the temperature reaction zone structure equation could be written in a different form, with the partial time derivative from the temperature total derivative moved to the right-hand side and grouped with the other unsteady terms. In that case, the non-dimensional equation equivalent to (3.5) would be

$$
\begin{aligned}
& \underbrace{\left(1-M^{2}\right) \frac{\gamma}{\gamma-1} w \frac{\partial \tilde{T}}{\partial \tilde{x}}}_{\text {spatial }}=\underbrace{\left(1-\gamma M^{2}\right) \tilde{Q} \tilde{k}(1-Z) \exp \left(-\frac{\tilde{E}_{a}}{\tilde{T}}\right)}_{\text {heat release }}+\underbrace{\frac{2}{\tilde{r}} \tilde{w}^{2}(\tilde{U}-\tilde{w})}_{\text {curvature }} \\
& +\underbrace{\tilde{w} \frac{\mathrm{d} \tilde{U}}{\mathrm{~d} \tilde{t}}-\tilde{w} \frac{\partial \tilde{w}}{\partial \tilde{t}}+\frac{1}{\tilde{\rho}} \frac{\partial \tilde{P}}{\partial \tilde{t}}-\left(1-M^{2}\right) \frac{\gamma}{\gamma-1} \frac{\partial \tilde{T}}{\partial \tilde{t}}}_{\text {unsteadiness }} .
\end{aligned}
$$

The left-hand side is now related to the spatial temperature gradient, rather than the total temperature gradient. This form allows direct evaluation of the quasi-steady assumption since omitting the unsteadiness expression gives the standard quasi-steady 

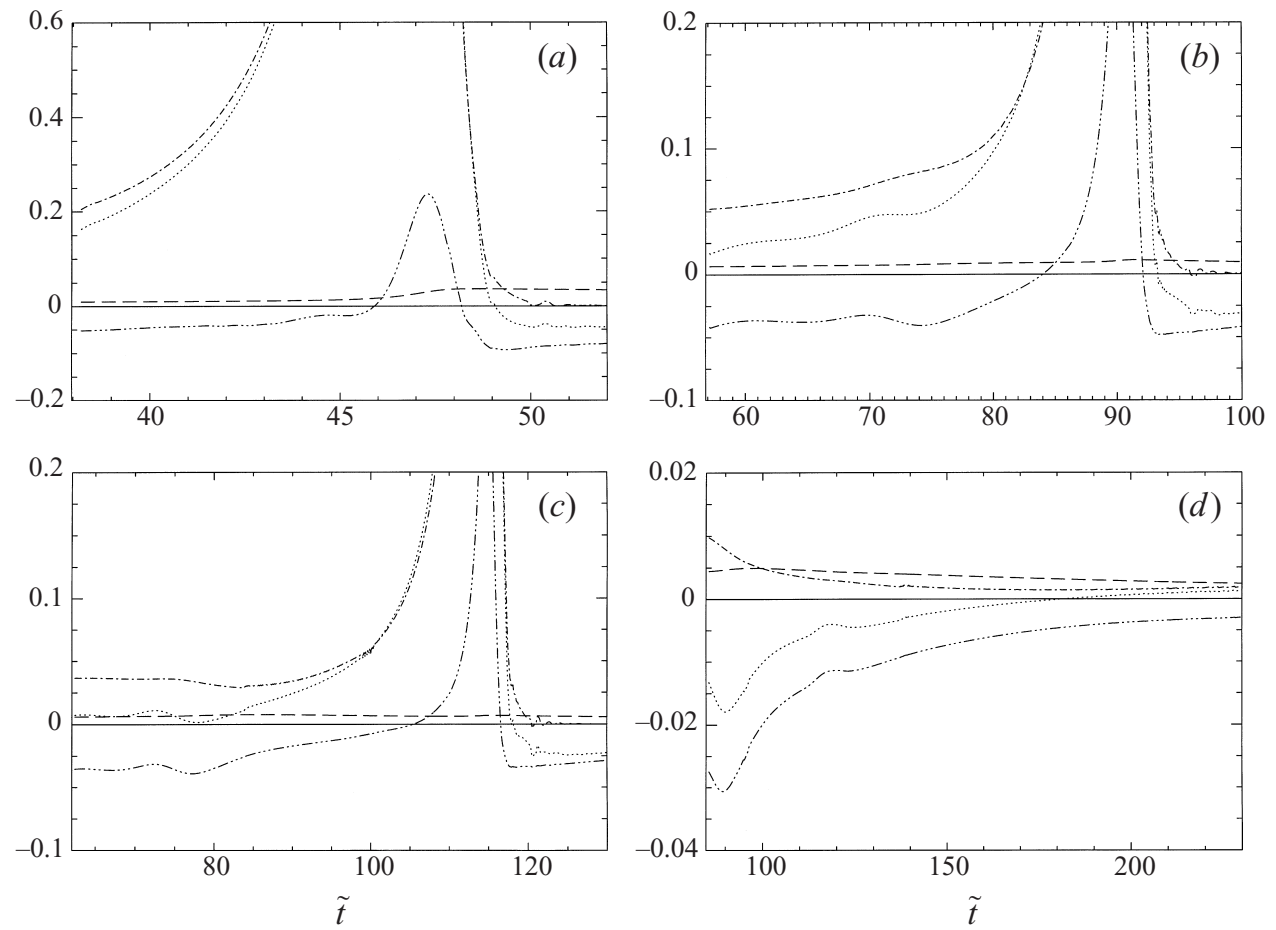

FIGURE 7. Terms in reaction-zone temperature equation (3.5) along the same particle paths as in figure 5 , for case A with $\widetilde{E}_{\text {source }}=160 \times 10^{6}: \cdots \cdots$, total temperature gradient; - $\cdot-\cdot$, heat release; --- , curvature; $-\cdots-\cdots$, unsteadiness. (a) Particle 1; (b) Particle 5; (c) Particle 6; $(d)$ Particle 10.

equation for the spatial temperature distribution, such as equation (A $2 a$ ) in He \& Clavin (1994).

In figure 8 , the terms in (3.7) are plotted along the same four particle paths that were shown in figure 7. Several observations can be made. First, the magnitude of the curvature term in the induction zone is still quite small compared to the unsteady terms. It is certainly not greater than the unsteady terms and hence a quasi-steady assumption is clearly erroneous for this flow. Secondly, all the terms on the right-hand side of the equation, including the unsteady terms, are actually of the same sign as the heat release in the induction zone. This makes a physical interpretation of the failure mechanism more difficult than with the reaction-zone structure equation (3.5). Finally, the unsteady terms show a steep variation through the induction zone, and no simplifying assumption regarding their behaviour is apparent. Contrast this with figure 7 where the unsteady terms were approximately constant in the induction zone, prior to failure. As will be seen in the next section, writing the equations in a form for which the unsteady terms are approximately constant is essential to our analysis as it reduces the governing PDE to an ODE. It is largely for this reason that we have chosen to use (3.5) in the analysis rather than (3.7).

It could also be argued that since equation (3.7) describes the spatial temperature gradient, its spatial variation should be examined, rather than the variation along a particle path. That is, it should be plotted in an Eulerian reference frame rather than a Lagrangian frame. In figure 9, the terms in this equation are plotted against $\tilde{x}$, the distance behind the leading shock, at several instants in time around the time of detonation failure. The results are qualitatively similar to figure 8 . The main 

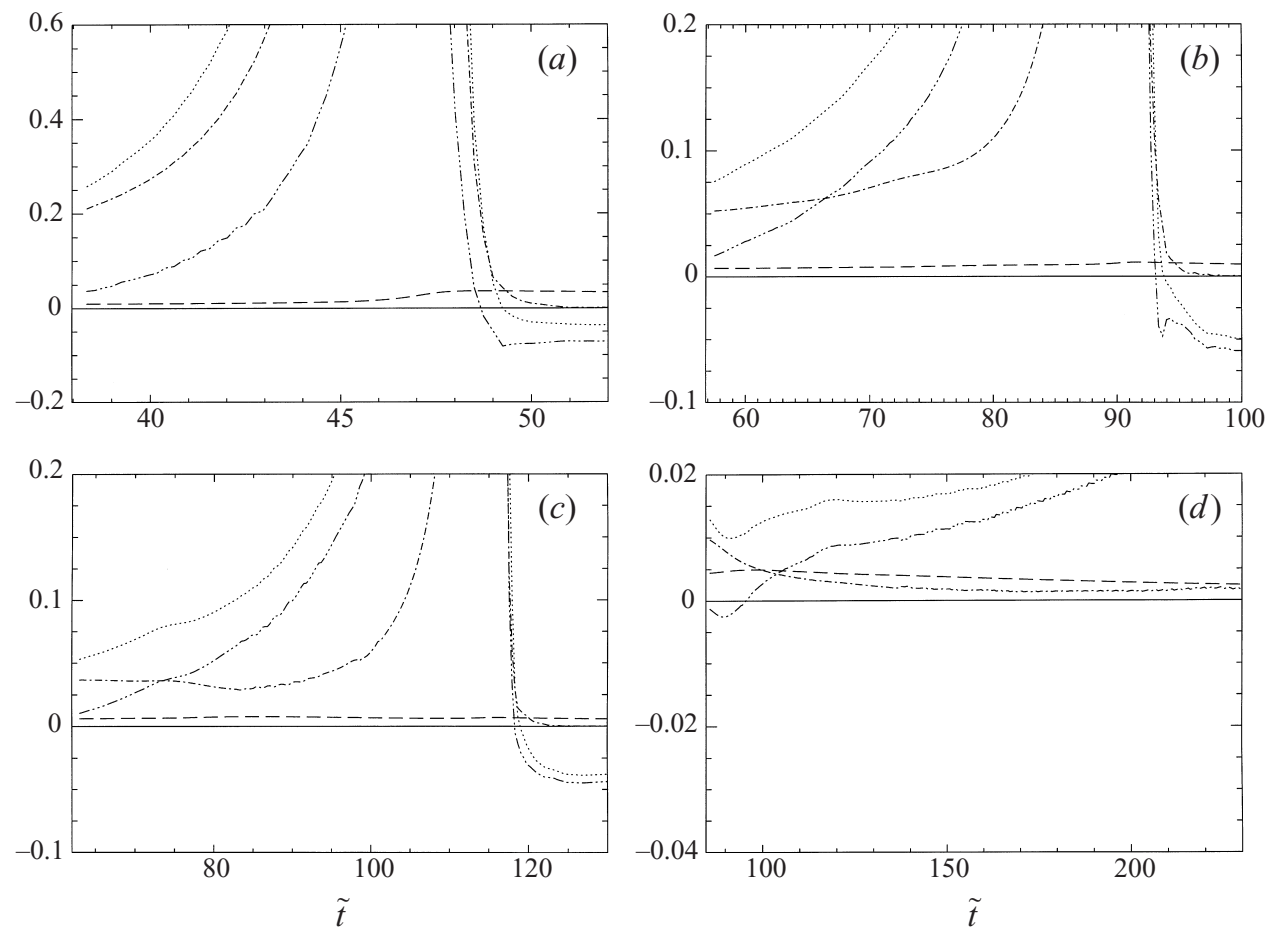

Figure 8. Terms in equation (3.7) along the same particle paths as in figure 5, for case A with $\tilde{E}_{\text {source }}=160 \times 10^{6}: \cdots \cdots$, spatial temperature gradient; $-\cdot-\cdot$, heat release; --- , curvature; $-\cdots+\cdots$, unsteadiness. (a) Particle 1; (b) Particle 5; (c) Particle 6; (d) Particle 10.

differences are at the late times in figures $9(c)$ and $9(d)$, where the unsteady terms are negative in the early part of the induction zone, and are of comparable magnitude to the curvature term. They are still not small compared to the curvature term, so even at these late times, the quasi-steady assumption is invalid. Other than this, all the conclusions of the previous paragraph apply.

We now turn our attention to the other computational case listed in table 1, case B. Figure 10 shows the velocity of the leading shock plotted against the shock radius, with several different source energies. The behaviour is more complex than in case A. For $\tilde{E}_{\text {source }} \leqslant 199 \times 10^{6}$, the detonation fails to initiate, with a monotonically decreasing shock strength. At $\tilde{E}_{\text {source }}=200 \times 10^{6}$, the detonation initiates, with a reinitiation explosion similar to that observed for the marginally supercritical source energy in case A. However, for $206 \times 10^{6} \leqslant \tilde{E}_{\text {source }} \leqslant 305 \times 10^{6}$, the detonation actually fails again, this time not with a monotonically decreasing shock strength, but with a single hump in the velocity profile. For $\tilde{E}_{\text {source }} \geqslant 306 \times 10^{6}$, the detonation initiates again, in a manner similar to that in case $\mathrm{A}$. The complex behaviour means we cannot identify a unique critical energy for this case. The behaviour can be summarized by plotting the location of the first two local maxima in the velocity profiles for several different source energies, as shown in figure 11. There are thus two critical energies, which we designate as $E_{c 1}$ and $E_{c 2}$, where $E_{c 1}<E_{c 2}$. An extensive scan of source energies in case $\mathrm{A}$ did not reveal the presence of a second critical energy, and we conclude that case A does have a unique critical energy.

A non-unique critical energy was also observed by Mazaheri (1997), although this 

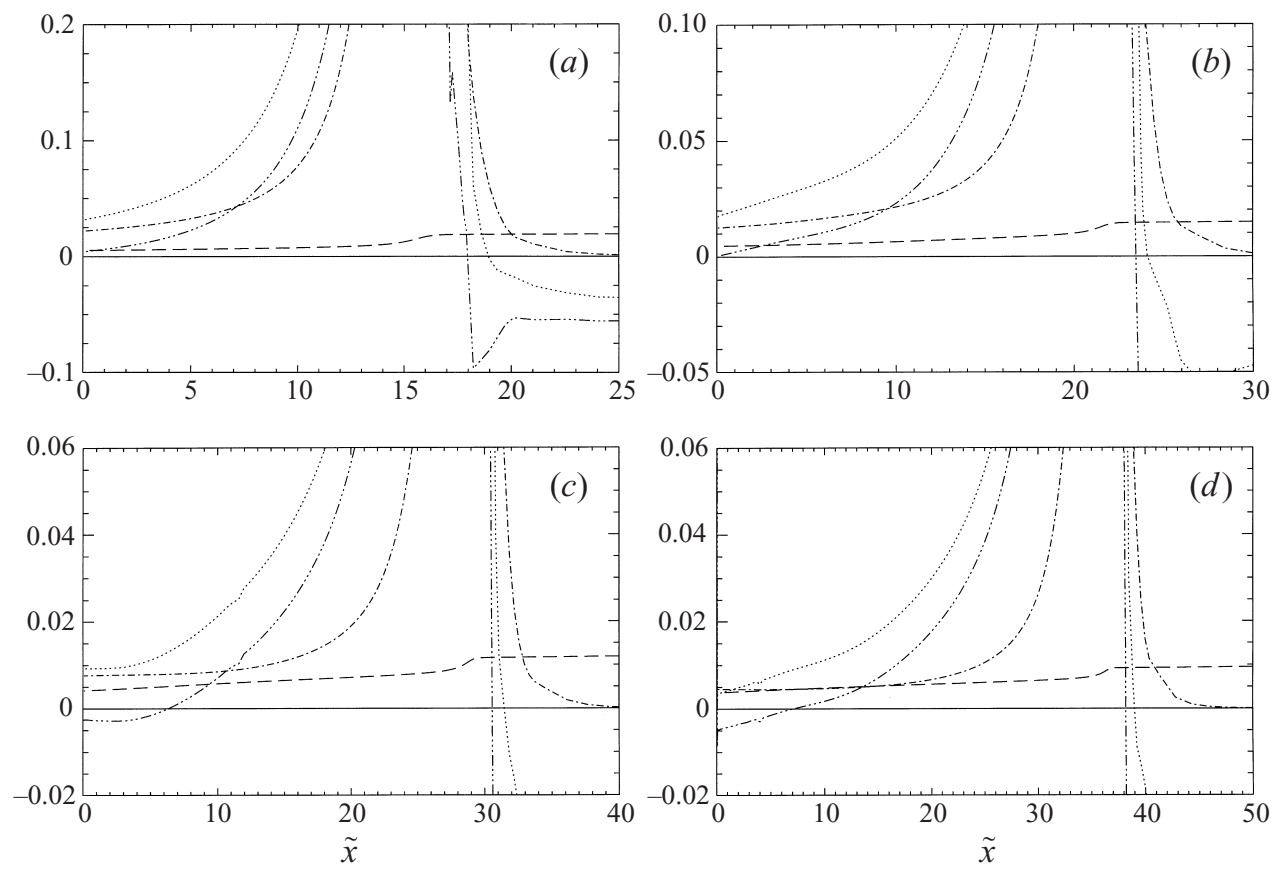

FIGURE 9. Spatial distribution of terms in equation (3.7), for case A with $\tilde{E}_{\text {source }}=160 \times 10^{6}$ : $\cdots \cdots$, spatial temperature gradient $;-\cdot-\cdot$, heat release; --- , curvature; $-\cdots-\cdots$, unsteadiness. (a) $\tilde{t}=70.5 ;$ (b) $\tilde{t}=80.3$; (c) $\tilde{t}=90.2 ;$ (d) $\tilde{t}=100.2$.

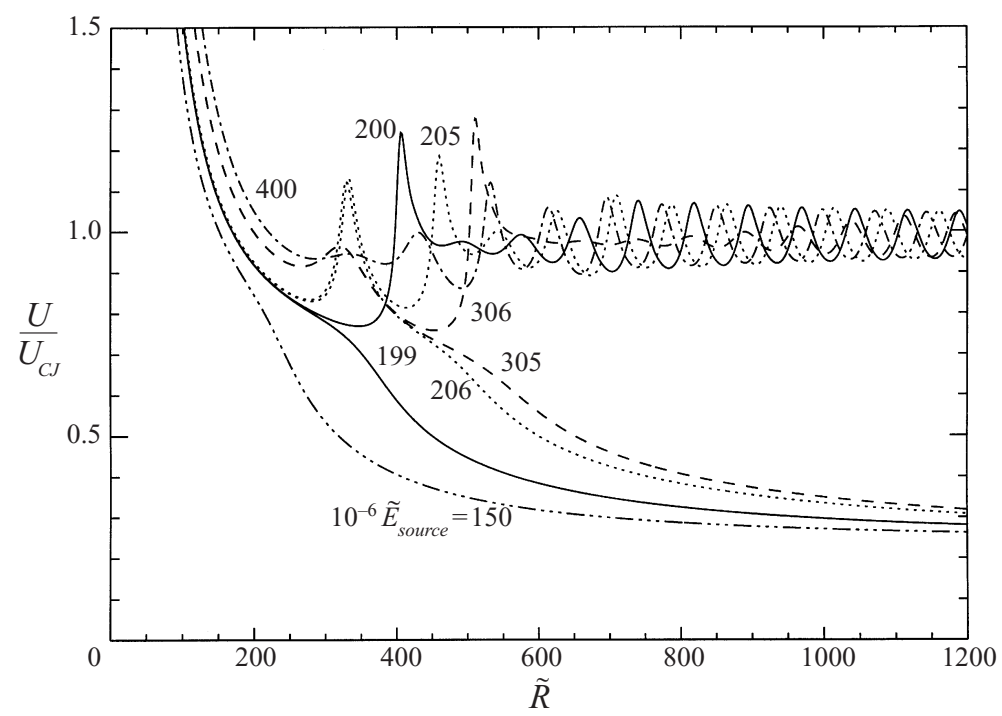

FIGURE 10. Leading shock velocity versus position for case B, with several different source energies. $\tilde{E}_{\text {source }}=150 \times 10^{6}, 199 \times 10^{6}, 200 \times 10^{6}, 205 \times 10^{6}, 206 \times 10^{6}, 305 \times 10^{6}, 306 \times 10^{6}, 400 \times 10^{6}$.

study was only performed at $\gamma=1.2$, where a second critical energy was found to exist for large activation energies. Our work shows that at larger $\gamma$, a second critical energy can exist even for lower activation energies near the neutral stability limit. This is an interesting result that challenges the very notion of critical energy, or at least, the 


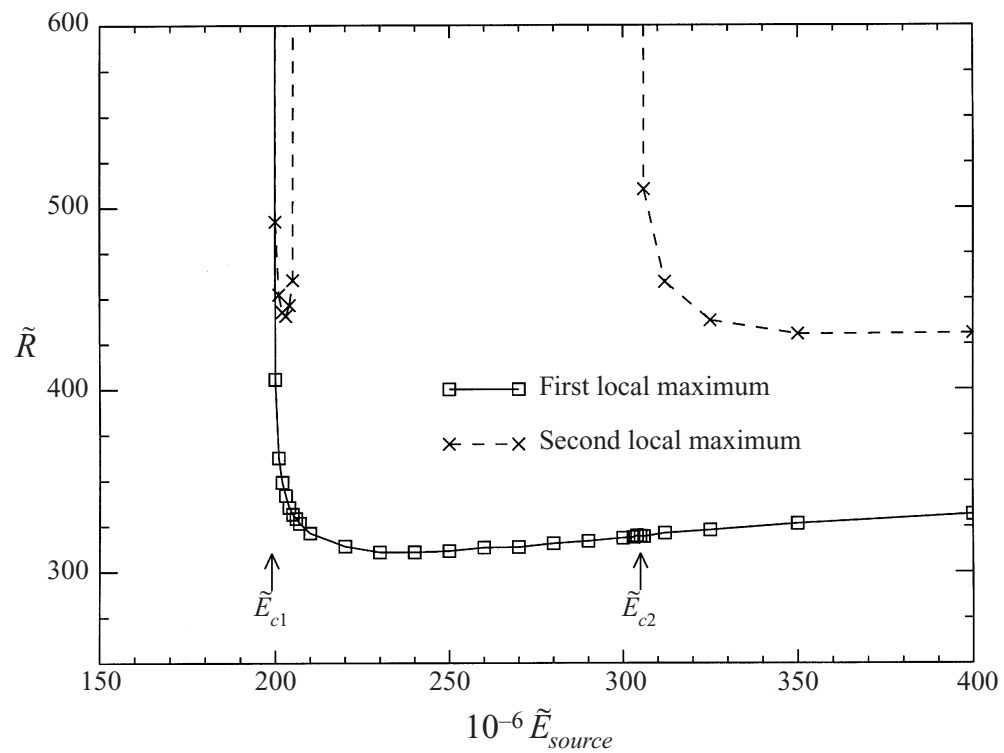

FIGURE 11. Location of first two local maxima in shock velocity profile for case B, as a function of source energy.

ability of the one-step reaction model to capture a critical energy. However, it is the subject of a whole research project in itself, and we will not pursue it further here. The velocity profiles around the first critical energy in case B appear to be very similar to those around the critical energy in case A. This suggests a universal behaviour at the lowest critical energy. For the remainder of this paper, including the development of a critical energy model equation, we will consider only this lower bound to the critical energy, where failure occurs with a monotonically decreasing shock strength. Hence, our model will at best give a lower-bound estimate of the critical energy. In figure 10, the two curves near the first critical energy, $\tilde{E}_{\text {source }}=199 \times 10^{6}$ and $200 \times 10^{6}$, begin to deviate significantly at about $\tilde{R}=280$, where $U / U_{C J}=0.8$. This is the critical point for the first critical energy, and it occurs at a similar shock velocity to that in case A.

The Lagrangian particle path information from figures 5 to 7 has been repeated in figures 12 to 14 for case B, with $\tilde{E}_{\text {source }}=199 \times 10^{6}$, a slightly subcritical source energy. Figure 12 shows the $r-t$ diagram with the paths of ten sample particles that cross the shock around the time of failure. The first few react rapidly while the last couple do not reach the reaction zone at all. The plot is similar to the earlier $r-t$ diagram for case A.

Figure 13 shows the evolution of the temperature along the same ten particle paths. Failure occurs more sharply than was observed in case A, with the reaction time growing rapidly around particle path 7 , and the post-shock temperature gradient decreasing quickly. Along the last few particle paths, there is a strong negative temperature gradient behind the shock and the reaction quenches, indicating detonation failure.

The terms in the temperature-reaction-zone structure equation (3.5) are plotted along four of the particle paths in figure 14. As before, the curvature term is small compared to the contributions from heat release and unsteadiness, at least prior to failure. For particles 3 and 5, both before failure, the unsteadiness is a negative forcing on the heat release term but is insufficient to prevent the reaction from proceeding. By 


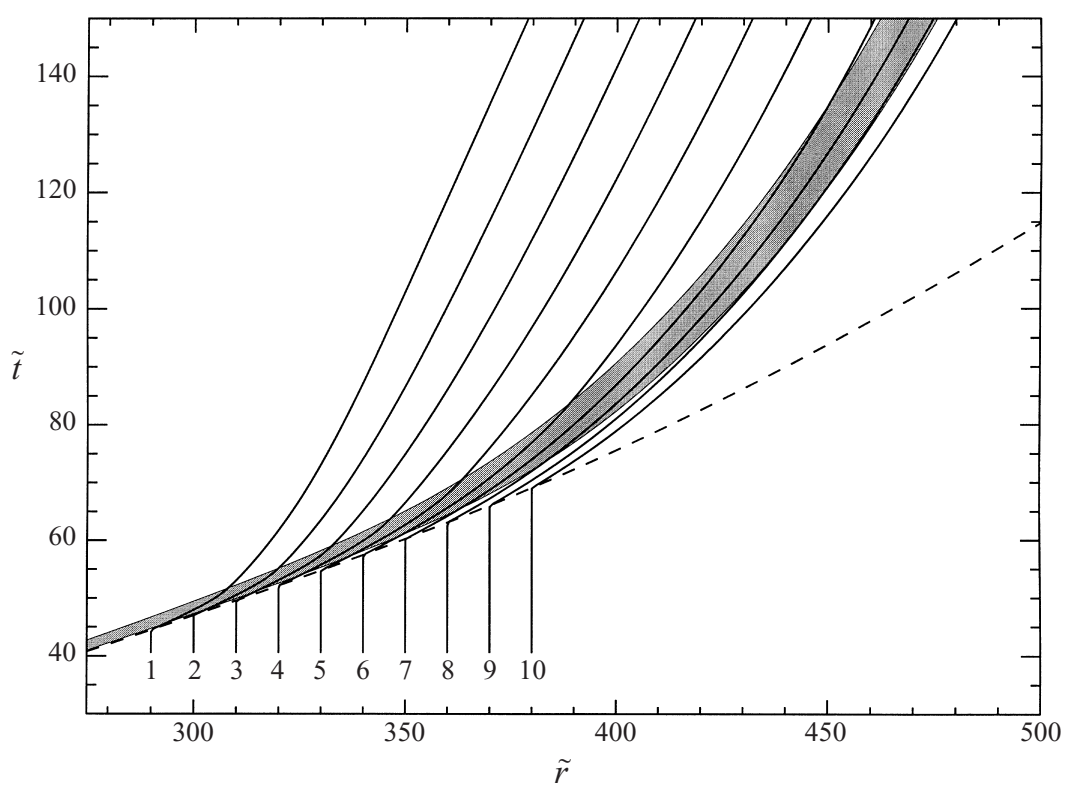

FIGURE 12. Particle paths for ten sample particles in case B, with $\tilde{E}_{\text {source }}=199 \times 10^{6}$. Shock (dashed line); $5 \%$ to $95 \%$ reaction (shaded region); particle paths (solid lines).

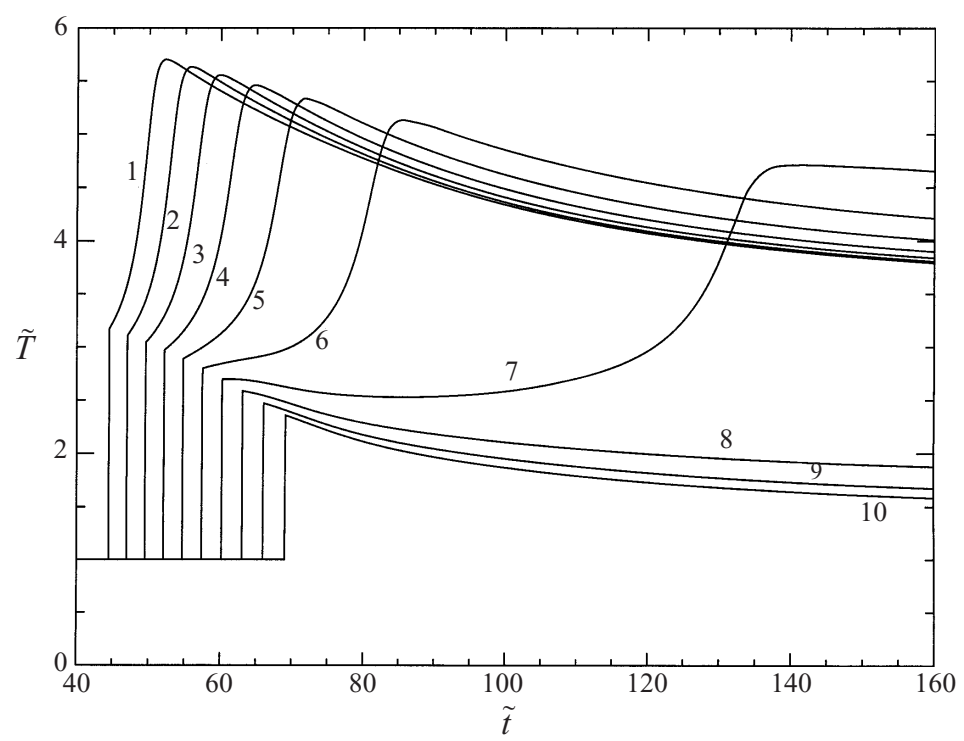

FIGURE 13. Temperature histories along the same ten particle paths as in figure 12, for case B with $\tilde{E}_{\text {source }}=199 \times 10^{6}$.

particle path 7, the magnitude of the unsteadiness is as great as the heat release term, and it significantly delays the reaction. It completely quenches the reaction by particle 9. As in figure 7, the unsteadiness expression is almost constant in the induction zone, for the particles prior to failure (particles 3 and 5). 

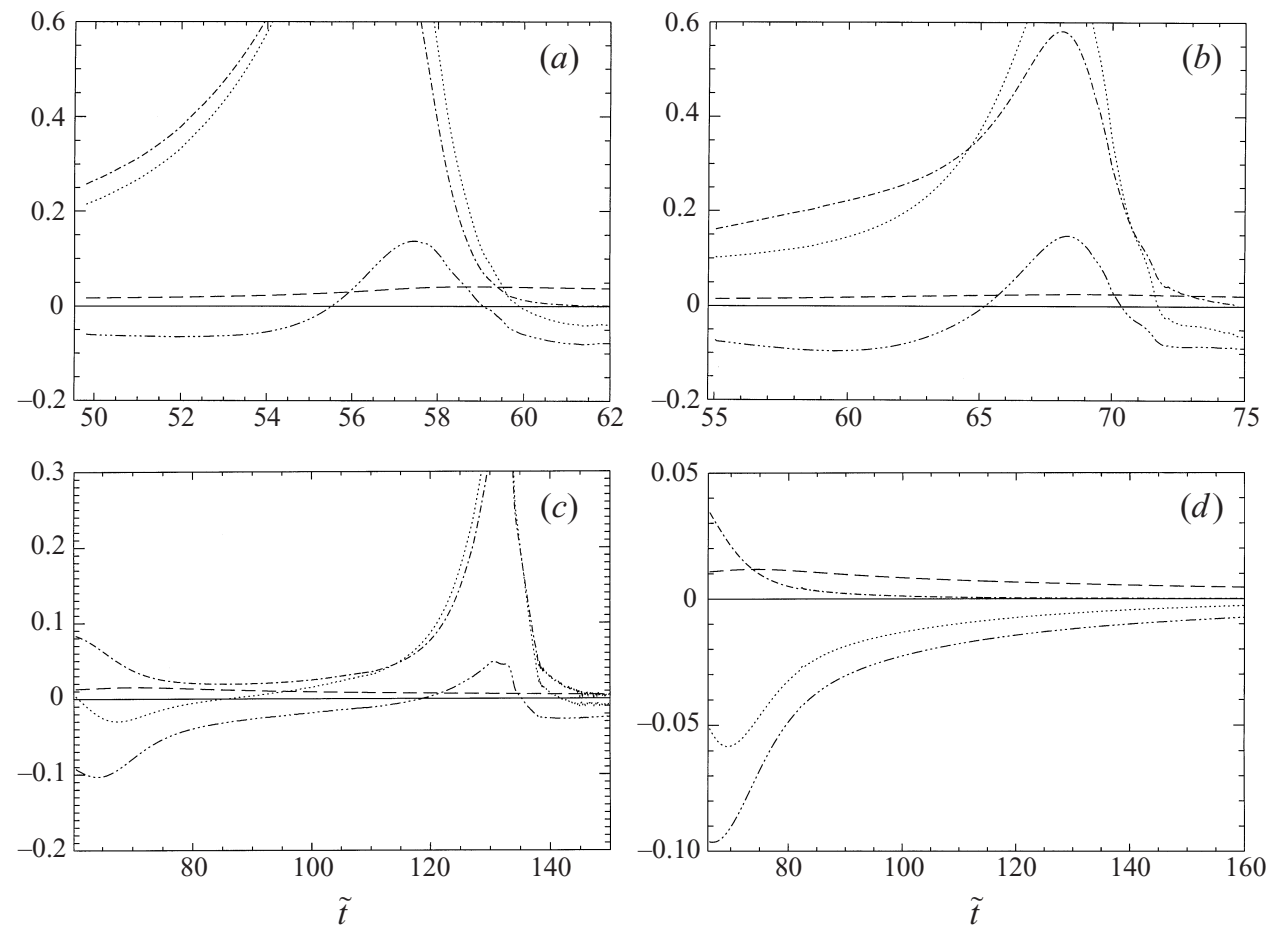

FIGURE 14. Terms in reaction-zone temperature equation (3.5) along the same particle paths as in figure 12 , for case B with $\tilde{E}_{\text {source }}=199 \times 10^{6}: \cdots \cdots$, total temperature gradient; $-\cdot \cdot-\cdot$, heat release; --- , curvature; $-\cdots+\cdots$, unsteadiness. (a) Particle 3; (b) Particle 5; (c) Particle $7 ;(d)$ Particle 9.

\section{Local initiation model}

In the previous section, the terms in the temperature-reaction-zone structure equation (2.13) were investigated along particle paths in the induction zones of near-critical blast initiations. It was found that the curvature term was negligible compared to the other terms. This same conclusion is obtained from an analytical consideration of the terms of the equation (see the Appendix). Additionally, the numerical simulations demonstrated that the unsteadiness expression was approximately constant. Thus, the unsteadiness expression can be approximated by its initial value on the particle path, that is, its value immediately after the shock at the time when the particle crosses the shock. Neglecting the curvature term, and setting the unsteadiness expression equal to its initial value immediately after the shock, (2.13) becomes

$$
\begin{aligned}
& \left(1-M^{2}\right) C_{P} \frac{\mathrm{D} T}{\mathrm{D} t} \\
& \quad=\left(1-\gamma M^{2}\right) Q k(1-Z) \exp \left(-\frac{E_{a}}{R_{g} T}\right)+\left(w_{s} \frac{\mathrm{d} U}{\mathrm{~d} t}-w_{s} \frac{\mathrm{d} w_{s}}{\mathrm{~d} t}+\frac{1}{\rho_{s}} \frac{\mathrm{d} P_{s}}{\mathrm{~d} t}\right)_{i},
\end{aligned}
$$

where subscript $s$ refers to conditions immediately after the shock, and subscript $i$ refers to the time $t_{i}$ when the particle under consideration initially crosses the shock. Note that the unsteadiness is now a constant forcing for a given particle, and we have reduced the equation from a PDE to an ODE. 
Recall the definition of the non-dimensional activation energy $\theta$ from (3.3). In particular,

$$
\theta_{i}=\frac{E_{a}}{R_{g} T_{s, i}}
$$

where $T_{s, i}$ is the post-shock temperature at time $t_{i}$. Note that $T_{s, i}$ and $\theta_{i}$ are functions only of the time $t_{i}$. Since $t_{i}$ is a constant for a given particle, then when applied along a particular particle path in (4.1), $T_{s, i}$ and $\theta_{i}$ will be constants. If the unsteadiness expression in (4.1) is of no greater magnitude than the heat release term, as was the case in the numerical simulations, then we can invoke standard large-activationenergy asymptotic expansions used to compute analytical induction times in the ZND model. Following this approach, we assume $\theta_{i} \gg 1$ and the temperature perturbation in the induction zone is small, $\delta T / T_{s, i}=O\left(1 / \theta_{i}\right)$. Then the following asymptotic expansion applies in the induction zone:

$$
\frac{T}{T_{s, i}}=1+\frac{1}{\theta_{i}} \hat{T}_{1}+O\left(\frac{1}{\theta_{i}^{2}}\right),
$$

where $\hat{T}_{1}$ is dimensionless and $O(1)$. Similarly, asymptotic expansions in Mach number and progress variable give

$$
\frac{M}{M_{s, i}}=1+O\left(\frac{1}{\theta_{i}}\right), \quad Z=O\left(\frac{1}{\theta_{i}}\right) .
$$

Using the above asymptotic expansions in (4.1) and retaining only the leading-order terms gives

$$
\left(1-M_{s, i}^{2}\right) C_{P} T_{s, i} \frac{1}{\theta_{i}} \frac{\mathrm{D} \hat{T}_{1}}{\mathrm{D} t}=\left(1-\gamma M_{s, i}^{2}\right) Q k \mathrm{e}^{\hat{T}_{1}-\theta_{i}}+\left(w_{s} \frac{\mathrm{d} U}{\mathrm{~d} t}-w_{s} \frac{\mathrm{d} w_{s}}{\mathrm{~d} t}+\frac{1}{\rho_{s}} \frac{\mathrm{d} P_{s}}{\mathrm{~d} t}\right)_{i} .
$$

Define a non-dimensional time by

$$
\zeta \equiv \frac{t-t_{i}}{\tau_{i}}
$$

where

$$
\tau=\frac{1}{k} \frac{1-M_{s}^{2}}{1-\gamma M_{s}^{2}} \frac{1}{\theta} \frac{C_{P} T_{s}}{Q} \mathrm{e}^{\theta},
$$

and $\tau_{i}$ is $\tau$ evaluated at time $t_{i}$. Then (4.2) reduces to

$$
\frac{\mathrm{D} \hat{T}_{1}}{\mathrm{D} \zeta}=\mathrm{e}^{\hat{T}_{1}}-\alpha_{i}
$$

where

$$
\alpha=-\frac{\theta \tau}{\left(1-M_{s}^{2}\right) C_{P} T_{s}}\left(w_{s} \frac{\mathrm{d} U}{\mathrm{~d} t}-w_{s} \frac{\mathrm{d} w_{s}}{\mathrm{~d} t}+\frac{1}{\rho_{s}} \frac{\mathrm{d} P_{s}}{\mathrm{~d} t}\right),
$$

and $\alpha_{i}$ is $\alpha$ evaluated at time $t_{i}$.

If $\alpha_{i}=0$, then (4.4) is identical to Frank-Kamenetskii's (1969) adiabatic homogeneous thermal explosion equation, under the approximation of large activation energy. With initial condition $\hat{T}_{1}=0$ when $\zeta=0$, it has solution

$$
\hat{T}_{1}=\ln \left(\frac{1}{1-\zeta}\right) \text {. }
$$

This 'explodes' $\left(\hat{T}_{1} \rightarrow \infty\right)$ at $\zeta_{\exp }=1$, so $\tau$ is the asymptotic induction time for a 
ZND detonation. If instead we consider $\alpha_{i}>0$, then (4.4) has solution

$$
\hat{T}_{1}=\ln \left\{\frac{\alpha_{i}}{1-\mathrm{e}^{\alpha_{i} \zeta}\left(1-\alpha_{i}\right)}\right\},
$$

and now

$$
\zeta_{\exp }=\frac{1}{\alpha_{i}} \ln \left(\frac{1}{1-\alpha_{i}}\right) .
$$

Note that $\zeta_{\text {exp }} \rightarrow \infty$ as $\alpha_{i} \rightarrow 1$, so in this model, a particle will undergo reaction in finite time provided $\alpha_{i}<1$ for that particle. We will refer to $\alpha$ as the initiation parameter, so the critical value of the initiation parameter is 1 .

The shock conditions are given by the perfect-gas jump conditions. Using the strong shock approximation for simplicity, these are

$$
\begin{array}{r}
P_{s}=\frac{2}{\gamma+1} \rho_{0} U^{2}, \quad \rho_{s}=\frac{\gamma+1}{\gamma-1} \rho_{0}, \quad w_{s}=\frac{\gamma-1}{\gamma+1} U, \\
T_{s}=\frac{P_{s}}{\rho_{s} R_{g}}=\frac{2(\gamma-1)}{(\gamma+1)^{2}} \frac{U^{2}}{R_{g}},
\end{array}
$$

where subscript 0 denotes the uniform conditions upstream of the shock. Substitution of (4.6) into (4.5) gives

$$
\alpha=6 \frac{\gamma-1}{\gamma+1} \theta \frac{\tau}{t_{d}},
$$

where $t_{d}$ is the characteristic shock decay time, defined by

$$
\frac{1}{t_{d}} \equiv-\frac{1}{U} \frac{\mathrm{d} U}{\mathrm{~d} t} .
$$

Then setting $\alpha=1$ in (4.7), the critical shock decay time is

$$
t_{d, c}=6 \frac{\gamma-1}{\gamma+1} \theta \tau .
$$

This equation is a local failure criterion as it predicts detonation success or failure based on a local analysis of the wave structure along a single particle path. In $\S 6$, the criterion will be utilized in a global analysis of the overall detonation initiation event in order to derive an equation for the critical energy. We refer to the model presented here as the critical decay rate (CDR) model.

Equation (4.9) indicates that the critical shock decay time is proportional to the detonation induction time, as expected from dimensional analysis. Since $6(\gamma-1) /(\gamma+$ 1) $\sim O(1)$ for typical values of $\gamma$, and $\theta \gg 1$, the equation also demonstrates that $t_{d, c} \gg \tau$. Failure occurs for any $t_{d} \leqslant t_{d, c}$, so unsteadiness can be important even when $t_{d} \gg \tau$, that is, when the characteristic time of evolution is much greater than the induction time. Contrast this with the statement of He \& Clavin (1994): 'When the characteristic time of evolution is much longer than the reaction time, unsteady terms may be neglected'. The authors used this statement as the basis for eliminating the unsteady terms in the governing equations at the outset of their analysis. The results of our simulations and the argument presented above show that their assumption is incorrect. It is only when $t_{d} \gg \theta \tau$ that unsteadiness can be neglected and the flow considered quasi-steady. 


\section{Validation of local initiation model with detailed kinetics}

Before considering the overall direct initiation event, an approximate numerical study of the local analysis can be made. Following a similar, although slightly different, reasoning as the previous section, imagine a hypothetical planar shock wave-reaction zone complex where the unsteady derivatives $\mathrm{d} U / \mathrm{d} t, \partial w / \partial t$ and $\partial P / \partial t$ can be approximated as constant along a particle path as the particle traverses the induction zone. The reaction-zone structure equations (2.6) then become

$$
\begin{aligned}
\eta \frac{\mathrm{D} w}{\mathrm{D} t} & =w \dot{\sigma}-M^{2}\left(\frac{\mathrm{d} U}{\mathrm{~d} t}\right)_{i}+\left(\frac{\mathrm{d} w_{s}}{\mathrm{~d} t}\right)_{i}-\frac{w}{\rho c^{2}}\left(\frac{\mathrm{d} P_{s}}{\mathrm{~d} t}\right)_{i}, \\
\eta \frac{\mathrm{D} \rho}{\mathrm{D} t} & =-\rho \dot{\sigma}+\frac{\rho w}{c^{2}}\left(\frac{\mathrm{d} U}{\mathrm{~d} t}\right)_{i}-\frac{\rho w}{c^{2}}\left(\frac{\mathrm{d} w_{s}}{\mathrm{~d} t}\right)_{i}+\frac{1}{c^{2}}\left(\frac{\mathrm{d} P_{s}}{\mathrm{~d} t}\right)_{i}, \\
\eta \frac{\mathrm{D} P}{\mathrm{D} t} & =-\rho w^{2} \dot{\sigma}+\rho w\left(\frac{\mathrm{d} U}{\mathrm{~d} t}\right)_{i}-\rho w\left(\frac{\mathrm{d} w_{s}}{\mathrm{~d} t}\right)_{i}+\left(\frac{\mathrm{d} P_{s}}{\mathrm{~d} t}\right)_{i} .
\end{aligned}
$$

We refer to these equations as the quasi-unsteady planar reaction zone structure equations. The term quasi-unsteady indicates that the unsteadiness is dealt with in an approximate manner which reduces the equations to ODEs.

For a system of ideal gases the derivatives $\mathrm{d} w_{s} / \mathrm{d} t$ and $\mathrm{d} P_{s} / \mathrm{d} t$ can be expressed in terms of $\mathrm{d} U / \mathrm{d} t$ as follows. The shock jump conditions are

$$
\rho_{0} U=\rho_{s} w_{s}, \quad P_{0}+\rho_{0} U^{2}=P_{s}+\rho_{s} w_{s}^{2}, \quad h_{0}+\frac{1}{2} U^{2}=h_{s}+\frac{1}{2} w_{s}^{2},
$$

where $h$ is the enthalpy. Differentiating these equations with respect to $U$ and using the ideal-gas caloric equation of state $\mathrm{d} h=C_{P} \mathrm{~d} T$, the derivatives $\mathrm{d} \rho_{s} / \mathrm{d} U, \mathrm{~d} w_{s} / \mathrm{d} U$ and $\mathrm{d} P_{s} / \mathrm{d} U$ can be solved. In particular,

$$
\begin{aligned}
& \frac{\mathrm{d} w_{s}}{\mathrm{~d} U}=\frac{\rho_{0}}{\rho_{s}}+\frac{M_{s}^{2}}{1-M_{s}^{2}}\left\{\left(\gamma_{s}+1\right) \frac{\rho_{0}}{\rho_{s}}-2 \gamma_{s}+\left(\gamma_{s}-1\right) \frac{\rho_{s}}{\rho_{0}}\right\}, \\
& \frac{\mathrm{d} P_{s}}{\mathrm{~d} U}=\rho_{0} U\left(2-\frac{\rho_{0}}{\rho_{s}}-\frac{\mathrm{d} w_{s}}{\mathrm{~d} U}\right) .
\end{aligned}
$$

Then the unsteady derivatives in (5.1) can be determined by

$$
\left(\frac{\mathrm{d} w_{s}}{\mathrm{~d} t}\right)_{i}=\left(\frac{\mathrm{d} w_{s}}{\mathrm{~d} U}\right)_{i}\left(\frac{\mathrm{d} U}{\mathrm{~d} t}\right)_{i}, \quad\left(\frac{\mathrm{d} P_{s}}{\mathrm{~d} t}\right)_{i}=\left(\frac{\mathrm{d} P_{s}}{\mathrm{~d} U}\right)_{i}\left(\frac{\mathrm{d} U}{\mathrm{~d} t}\right)_{i} .
$$

This reduces the unsteady derivatives in the reaction-zone structure equations to a single parameter $(\mathrm{d} U / \mathrm{d} t)_{i}$, which can be specified in the form of a characteristic shock decay time $t_{d}$ as in (4.8).

Since the reaction-zone structure equations have been reduced to simple ODEs, it is numerically inexpensive to integrate them for real gas systems. This has been done for $\mathrm{H}_{2}$-air, $\mathrm{H}_{2}-\mathrm{O}_{2}$ and $\mathrm{C}_{2} \mathrm{H}_{4}$-air systems for various equivalence ratios, with detonation waves at $\mathrm{CJ}$ velocity. The detailed reaction mechanism used was the hydrocarbon mechanism from Appendix A of Miller \& Bowman (1989), with nitrogen chemistry removed and carbon chemistry also removed for the $\mathrm{H}_{2}$ systems. Realistic thermochemistry was attained from the CHEMKIN package (Kee, Rupley \& Miller 1989). The solution procedure first involved computing the CJ velocity using the chemical equilibrium code STANJAN (Reynolds 1986). The root finder ZEROIN (Shampine \& Watts 1970) was then used to find the post-shock state, the initial conditions for the reaction-zone structure equations. For a given first guess of the critical shock decay time, the equations were integrated forward in time using the backward differencing 


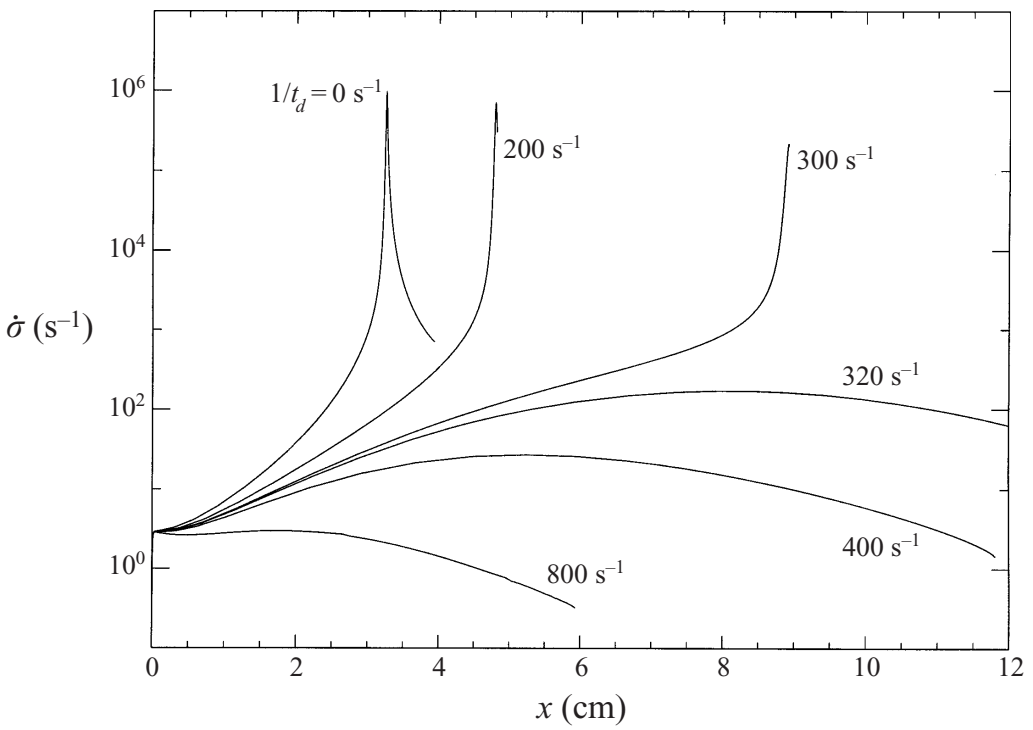

FIGURE 15. Total thermicity versus distance downstream of the shock, from quasi-unsteady calculations, for $15 \% \mathrm{H}_{2}$ in air and various shock decay times.

stiff ODE solver DEBDF (Shampine \& Watts 1979). An indication of whether or not the reaction was quenched by the applied degree of unsteadiness $t_{d}$ could be gauged from the total thermicity $\dot{\sigma}$. Figure 15 shows the variation of total thermicity through the reaction zone for $15 \%$ by volume $\mathrm{H}_{2}$ in air, with various values of the shock decay time. It is clear from this plot that at quenching the thermicity fails to develop a sharp peak and the maximum greatly decreases. The variation of maximum thermicity $\dot{\sigma}_{\max }$ with shock decay time is shown in figure 16 . A somewhat arbitrary choice was made to define detonation failure as the point where the maximum thermicity dropped to $1 \%$ of its value at steady flow $\left(1 / t_{d}=0\right)$. As demonstrated in figure 16 , the determination of the critical shock decay time is not very sensitive to the cutoff value chosen.

The numerically computed critical shock decay times were compared with the theoretical predictions from (4.9). The parameters used in (4.9) were determined as follows. The planar ZND induction time $\tau$ for the CJ wave was determined by integration of the planar steady form of the reaction-zone structure equations $(2.6 a)$ (2.6c), using the detailed reaction mechanism, behind a shock travelling at CJ velocity. The induction time was identified as the point of maximum temperature gradient $\mathrm{d} T / \mathrm{d} t$. The equivalent value of $\gamma$ chosen for (4.9) was determined by matching the post-shock temperature in the detailed reaction system to that in the one-step model, as the temperature is the most important state quantity to represent correctly in the induction zone. For the constant- $\gamma$ model, the exact temperature ratio across the shock is given by

$$
\frac{T_{s}}{T_{0}}=\frac{\left\{2 \gamma M_{0}^{2}-(\gamma-1)\right\}\left(\gamma-1+2 / M_{0}^{2}\right)}{(\gamma+1)^{2}} .
$$

This equation was solved to determine the equivalent constant value of $\gamma$ for the detailed reaction system at a specified free-stream Mach number and shock temperature ratio. The estimated value of $\theta$ for the detailed reaction system was determined by a method described in Shepherd (1986). This method proceeds by considering approxi- 


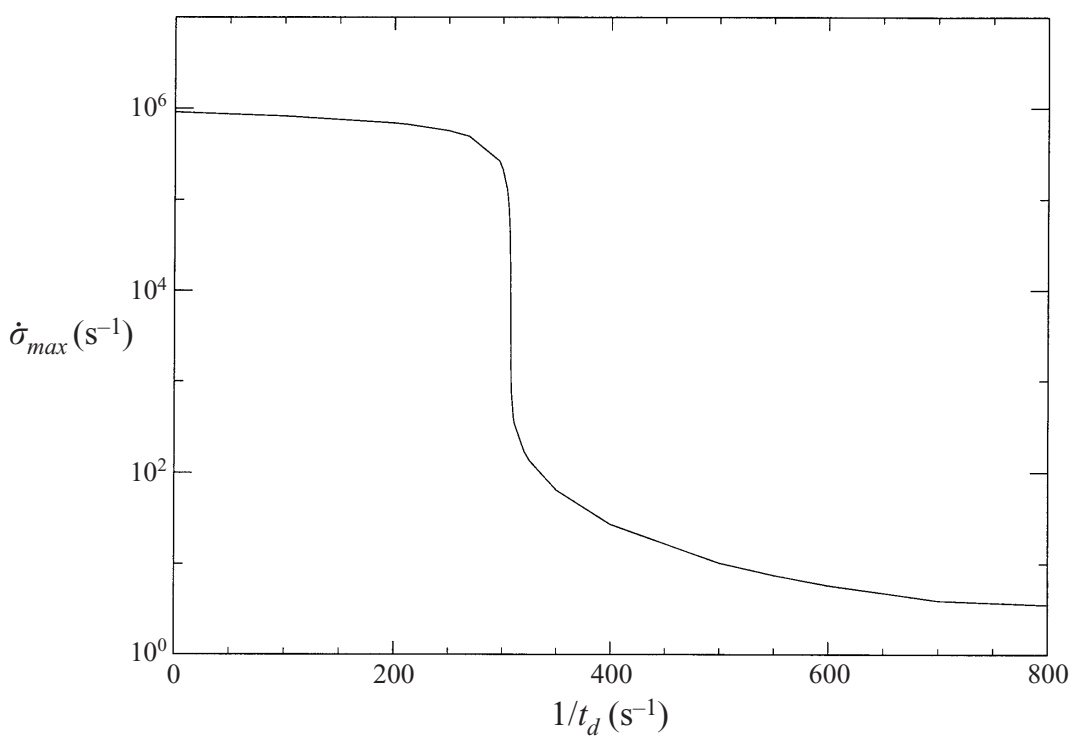

FIGURE 16. Maximum thermicity versus characteristic shock decay time, from quasi-unsteady calculations, for $15 \% \mathrm{H}_{2}$ in air.

mating a system of reactions by a single global rate. The conventional approximation to induction time corresponding to a global rate is

$$
\tau=C[\text { fuel }]_{i}^{a}[\text { oxidizer }]_{i}^{b} \exp \left(\frac{E_{a}}{R_{g} T_{i}}\right),
$$

where $C$ is a pre-exponential constant, the square brackets indicate initial concentrations, $a$ and $b$ are empirical constants, and $T_{i}$ is the initial temperature, in our case the post-shock temperature $T_{s}$. If we differentiate this expression with respect to $T_{i}$, holding the initial density and mass fractions constant, then the initial concentrations will remain constant, and $\theta$ will be given by

$$
\theta=\frac{E_{a}}{R_{g} T_{i}}=-\left.\frac{T_{i}}{\tau} \frac{\partial \tau}{\partial T_{i}}\right|_{\rho_{i}, \boldsymbol{y}_{i}} .
$$

This enables the determination of the global parameter $\theta$ by carrying out constantvolume simulations to find $\tau$, using a detailed reaction mechanism and realistic thermochemistry. The derivative was computed numerically by perturbing the initial temperature $T_{i}=T_{s}$ while holding the initial density $\rho_{i}=\rho_{s}$ and the initial mass fractions constant. The induction time $\tau$ was identified as the point of maximum temperature gradient $\mathrm{d} T / \mathrm{d} t$. The same reaction mechanism was used as in the quasiunsteady and ZND simulations described previously.

The numerically computed critical shock decay times are plotted with the CDR model predictions from (4.9) in figure 17. For each fuel-oxidizer mixture shown, the induction time varies by several orders of magnitude over the range of equivalence ratios. The critical shock decay time essentially follows the same trend, so to best compare the numerical and theoretical values this dominant trend has been removed by normalizing the critical shock decay times by the induction times. The theoretical predictions agree reasonably well with the numerical data in all three cases, although in general the theory underpredicts the critical shock decay time by as much as $40 \%$. 

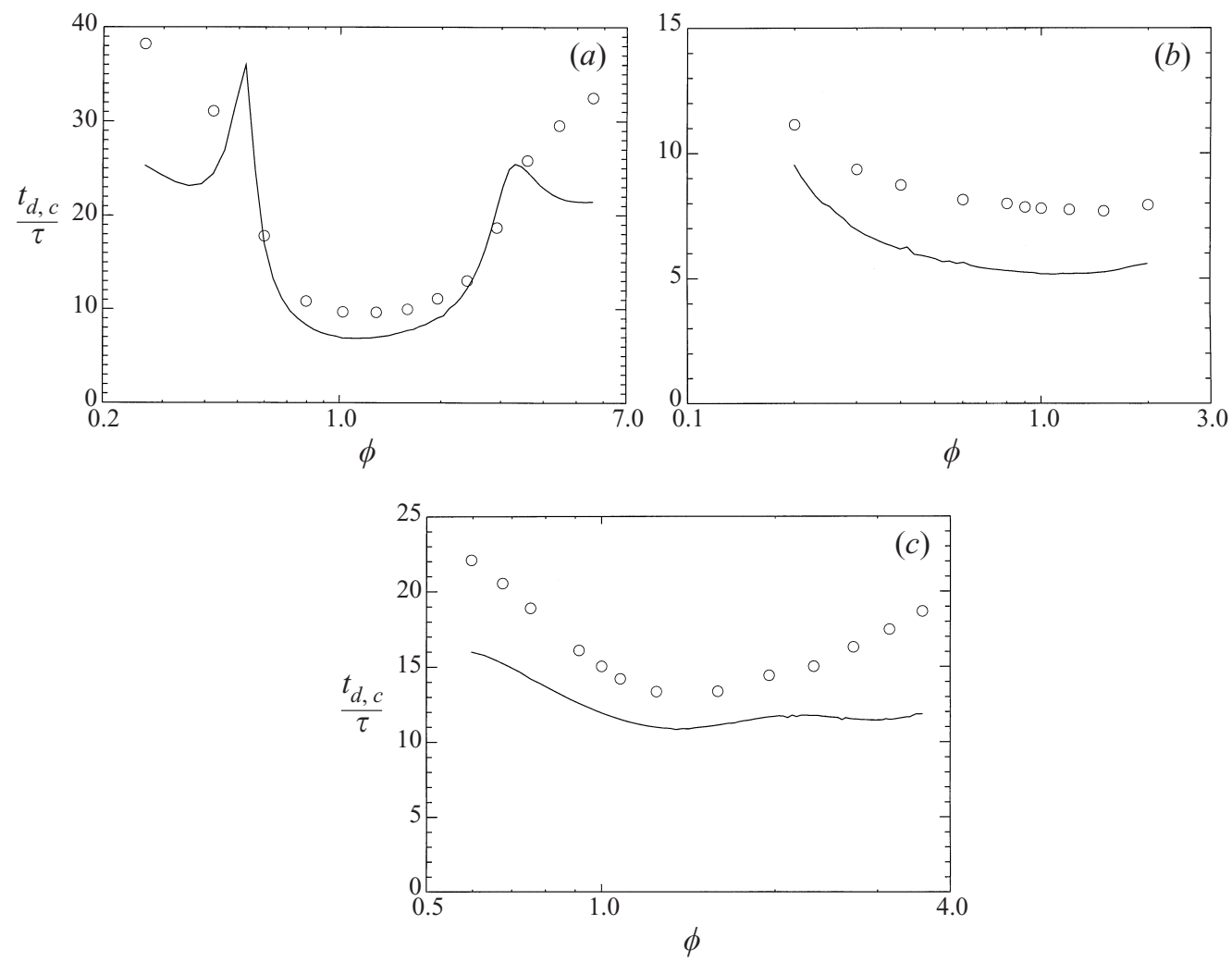

FIGURE 17. Critical shock decay time versus equivalence ratio. Lines: critical decay rate model, (4.9); symbols: numerical results from quasi-unsteady calculations with (5.1). (a) $\mathrm{H}_{2}$-air; (b) $\mathrm{H}_{2}-\mathrm{O}_{2}$; (c) $\mathrm{C}_{2} \mathrm{H}_{4}$-air.

We believe this is quite satisfactory considering the crude approximations made in using $(a)$ the one-step mechanism to simulate the real chemical system, $(b)$ the large activation energy asymptotics, and $(c)$ the strong shock assumption. Note from (4.9) that the theoretical value of $t_{d, c} / \tau$ is proportional to $\theta$. The unusual behaviour of $\theta$ near the lean and rich ends of the $\mathrm{H}_{2}$-air system has been previously documented (Shephard 1986), and is evidenced in the theoretical curve of figure 17(a). The same trend does not appear in the quasi-unsteady calculations.

\section{Global initiation criterion}

In $\S 4$, an initiation criterion was developed based on a local analysis of the reactionzone structure. To convert this criterion into a useful predictive formula for the critical energy, it must be applied to the global initiation event. A priori knowledge of the approximate blast wave velocity profile is required, so that the shock decay rate may be computed in terms of the controlling parameters of the problem.

The simplest choice, and that used by most previous workers on the blast initiation problem, is the Taylor-Sedov similarity solution for a non-reacting strong point blast (Taylor 1950; Sedov 1959). The equations for this point-blast theory (PBT) were listed in $\S 3.1$ for the spherical case $(j=2)$. In more generality, the blast wave profile is 
given by

$$
\begin{aligned}
R & =\left(\frac{E_{\text {source }}}{A_{j} \rho_{0}}\right)^{1 /(j+1)}\left(\frac{2}{j+3} \frac{1}{U}\right)^{2 /(j+1)}=\left(\frac{E_{\text {source }}}{A_{j} \rho_{0}}\right)^{1 /(j+3)} t^{2 /(j+3)}, \\
t & =\left(\frac{E_{\text {source }}}{A_{j} \rho_{0}}\right)^{1 /(j+1)}\left(\frac{2}{j+3} \frac{1}{U}\right)^{(j+3) /(j+1)},
\end{aligned}
$$

where $E_{\text {source }}$ is the initial energy release for spherically symmetric flow, the energy release per unit length for cylindrically symmetric flow, or the energy release per unit area for planar flow. $A_{j}$ is the energy integral constant, and is a function of $j$ and $\gamma$. A correlation for the spherical case $(j=2)$ was given in (3.4).

However, the PBT does not account for the significant effect of chemical energy release, and to a lesser extent, finite back pressure. Korobeinikov (1968) proposed a method for including the effect of chemical energy release on the analytical blast wave profile, using a linearization of the reacting flow governing equations about the non-reacting PBT solution. This linearization results in the following solution:

$$
\begin{aligned}
R & =\left(\frac{E_{\text {source }}}{A_{j} \rho_{0}}\right)^{1 /(j+1)}\left(\frac{2}{j+3} \frac{1}{U}\right)^{2 /(j+1)} \exp \left\{\frac{B_{j} Q}{(j+1) U^{2}}\right\}, \\
t & =\left(\frac{E_{\text {source }}}{A_{j} \rho_{0}}\right)^{1 /(j+1)}\left(\frac{2}{j+3} \frac{1}{U}\right)^{(j+3) /(j+1)}\left\{1+\frac{(j+2)(j+3)}{(j+1)(3 j+5)} \frac{B_{j} Q}{U^{2}}\right\},
\end{aligned}
$$

where the last factor in each equation is the reacting flow correction. $B_{j}$ is another energy integral constant, and is again a function of $j$ and $\gamma$. Korobeinikov (1991) lists values of $B_{j}$ for $j=0,1,2$ and various values of $\gamma$. A fit of this data in the spherical case $(j=2)$ gives

$$
B_{2}=4.1263(\gamma-1)^{1.2530+0.14936 \log _{10}(\gamma-1)},
$$

accurate to $0.29 \%$ in the range $1.2 \leqslant \gamma \leqslant 2.0$.

The linearized solution given by (6.1) should strictly only be valid before the blast wave has decayed to the CJ velocity. However, in practice, it is a good approximation for a considerably longer time, at least in the case of initiation failure. This is evidenced in figure 18 where the numerical blast wave velocity profile of a near-critical initiation event, case A with $\tilde{E}_{\text {source }}=166 \times 10^{6}$, is plotted with the corrected PBT profile. While there is some discrepancy between the curves, the discrepancy gets no worse at the lower shock velocities. Hence, the theoretical curve seems to be applicable down to at least $U=0.7 U_{C J}$. For comparison, the standard non-reacting PBT profile is also plotted in this figure. Clearly, the corrected PBT curve is a much better approximation to the numerical curve. The corrected PBT curve could be shifted even closer to the numerical curve by additionally considering the correction due to finite back pressure, but the correction is very small for the regime shown in figure 18 , so the improvement would be negligible.

The characteristic shock decay time $t_{d}$ for the corrected PBT can be found by differentiating $(6.1 b)$, giving

$$
\begin{aligned}
t_{d} & =-\frac{U}{\mathrm{~d} U / \mathrm{d} t} \\
& =\frac{j+3}{j+1}\left(\frac{2}{j+3}\right)^{(j+3) /(j+1)}\left(\frac{E_{\text {source }}}{A_{j} \rho_{0}}\right)^{1 /(j+1)}\left(1+\frac{j+2}{j+1} \frac{B_{j} Q}{U^{2}}\right) U^{-(j+3) /(j+1)} .
\end{aligned}
$$




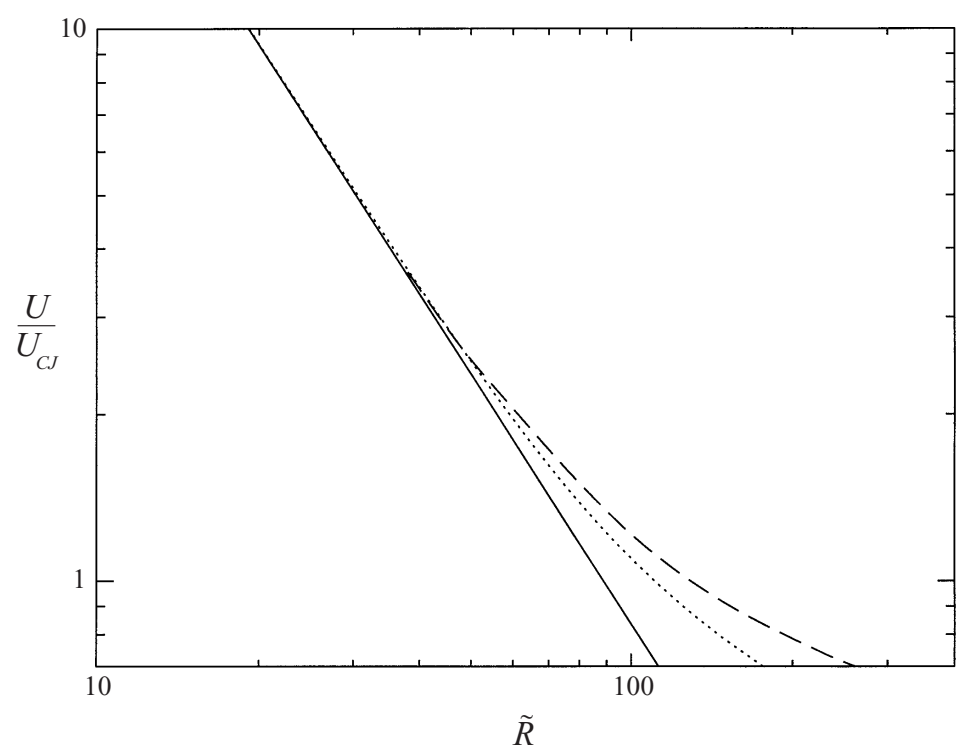

FIGURE 18. Shock velocity profiles for case A with $\tilde{E}_{\text {source }}=166 \times 10^{6}$. - , Taylor-Sedov non-reacting point blast theory (PBT); $\cdots \cdots$, PBT with linearized reacting flow correction; --- , numerical simulation.

It must now be decided at what point in the blast wave profile to evaluate $t_{d}$ and check against the failure criterion (4.9). The simplest choice is to evaluate the model at $U=U_{C J}$, since failure is likely to occur in that vicinity. However, closer examination of the numerical simulation results in figures 3 and 10 reveals that failure actually occurs somewhat below $U_{C J}$ in the critical initiations. Denote the velocity of the leading shock at failure as $U_{*}$. We will discuss the selection of $U_{*}$ later in this section. Define $\theta_{*}$ and $\tau_{*}$ as the values of $\theta$ and $\tau$ when $U=U_{*}$. Then setting $E_{\text {source }}=E_{c}$ when $t_{d}=t_{d, c}$, and combining (6.2) with (4.9), gives the critical energy:

$$
E_{c}=A_{j}\left(6 \frac{j+1}{j+3} \frac{\gamma-1}{\gamma+1} \theta_{*}\right)^{j+1}\left(\frac{j+3}{2}\right)^{j+3}\left(1+\frac{j+2}{j+1} \frac{B_{j} Q}{U_{*}^{2}}\right)^{-(j+1)} \rho_{0} U_{*}^{j+3} \tau_{*}^{j+1} .
$$

This is the final model equation for the critical energy, under the assumptions of the critical decay rate $(\mathrm{CDR})$ model. For spherically symmetric flow $(j=2),(6.3)$ gives

$$
E_{c}=4.56 \times 10^{3} A_{2}\left(\frac{\gamma-1}{\gamma+1} \theta_{*}\right)^{3}\left(1+\frac{4}{3} \frac{B_{2} Q}{U_{*}^{2}}\right)^{-3} \rho_{0} U_{*}^{5} \tau_{*}^{3} .
$$

Using the non-dimensional notation of $\S 3,(6.4)$ becomes

$$
\tilde{E}_{c}=4.56 \times 10^{3} A_{2}\left(\frac{\gamma-1}{\gamma+1} \theta_{*}\right)^{3}\left(1+\frac{4}{3} \frac{B_{2} \tilde{Q}}{\tilde{U}_{*}^{2}}\right)^{-3} \tilde{U}_{*}^{5} \tilde{\tau}_{*}^{3}
$$

This equation can be used to predict critical energies for the one-step model used in the numerical simulations. The selection of $\tilde{U}_{*}$ can be made empirically, by examining the shock velocity profiles in the numerical simulations. The failure point is identified as the point where the profiles of the marginally subcritical and marginally supercritical initiation energies start to deviate significantly. This was done in $\S 3.2$, where it was determined that $U_{*}=0.75 U_{C J}$ for case A and $U_{*}=0.8 U_{C J}$ for the first critical energy 


\begin{tabular}{ccc} 
& \multicolumn{2}{c}{ Critical energy } \\
\cline { 2 - 3 } Case & Model & Numerical \\
A & $34.6 \times 10^{6}$ & $166 \times 10^{6}$ \\
B & $52.3 \times 10^{6}$ & $199 \times 10^{6}$
\end{tabular}

TABLE 2. Comparison between model predictions and numerical results for critical energy.

in case B. Since $U_{*}<U_{C J}$ there is no steady ZND solution, so the induction time $\tau_{*}$ must be computed by some method other than a ZND calculation. In this work, we have used a constant-pressure reaction behind a shock travelling at velocity $U_{*}$, and identified the induction time as the point of maximum temperature gradient. A constant-pressure assumption gives induction times in very close agreement with the ZND model. This can be verified by considering the one-step reaction model. Under the assumption of constant pressure, the asymptotic induction time is given by

$$
\tau=\frac{1}{k} \frac{1}{\theta} \frac{C_{P} T_{s}}{Q} \mathrm{e}^{\theta}
$$

Comparing this with (4.3), the ratio of the asymptotic induction time in the constantpressure model to that in the ZND model is

$$
\frac{1-\gamma M_{s}^{2}}{1-M_{s}^{2}}
$$

In the strong shock limit, this ratio is

$$
\frac{\gamma(3-\gamma)}{\gamma+1}
$$

and for $\gamma$ not much larger than 1 , this ratio is very close to 1 .

The critical energy predictions of (6.5) are listed in table 2 for the two computational cases. They are compared with the values determined directly from the numerical simulations, where the first critical energy $\tilde{E}_{c 1}$ is listed for case B. The model underpredicts the critical energy by a factor of 4 to 5 . Some disagreement between the model and the numerics was expected, as the numerical simulations used a relatively low activation energy that produced a reaction zone with no clearly identifiable induction zone. The model assumes an ideal asymptotic induction zone, and this is closer to what is observed in real gas systems. Hence the numerical simulations were intended mainly for qualitative validation of the model, rather than quantitative comparison.

For practical application of the critical energy equation (6.3) in real gas detonations, various parameters need to be determined. The value of $\theta_{*}$ can be determined by the method described in $\S 5$, with a constant-volume reaction behind a shock travelling at velocity $U_{*}$. Similarly, the value of $\gamma$ is determined by matching the shock temperature ratio at the shock velocity $U_{*}$. The induction times $\tau_{*}$ are computed from a constantpressure calculation, as for the one-step model earlier in this section. The heat of reaction $Q$ is defined as the difference between the heats of formation of the reactant and product, where the heats of formation are the enthalpies of the reactant mixture and the equilibrium product mixture, with each at a standard reference temperature of $300 \mathrm{~K}$. The equilibrium product composition is taken from the constant-pressure reaction calculation behind a shock travelling at velocity $U_{*}$. 

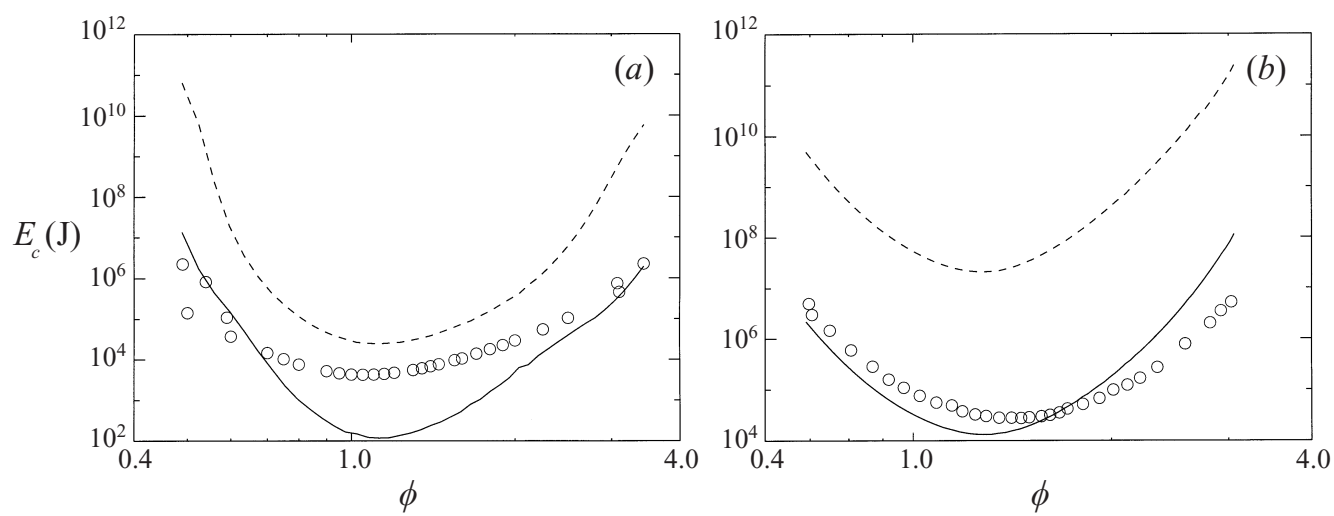

FIGURE 19. Comparison between theory and experiment for critical energy versus equivalence ratio: _ , critical decay rate model, (6.4); - - - , critical curvature model (He \& Clavin 1994), (7.3); ○, experiment (Benedick et al. 1986). (a) $\mathrm{H}_{2}$-air; (b) $\mathrm{C}_{2} \mathrm{H}_{4}$-air.

This just leaves the specification of the shock velocity $U_{*}$ where the critical decay rate model will be applied. Without the benefit of numerical results for each real gas detonation, a theoretical prescription is necessary. For this paper, we have assumed $U_{*}=U_{c}$, where $U_{c}$ is the shock velocity corresponding to the critical radius $R_{c}$ for a slightly curved, quasi-steady detonation. Although failure occurs at a shock radius smaller than the critical radius, as shown in the following section, this quasisteady solution appears to be the attractor for successfully initiated detonations with a marginally supercritical energy (He \& Clavin 1994). So $U_{c}$ will be a reasonable estimate for the shock velocity in the critical region of the flow. We have taken the following expression for the velocity $U_{c}$, derived from a square-wave detonation model (He \& Clavin 1994):

$$
U_{c}=U_{C J}\left(1-\frac{1}{2 \theta_{C J}}\right) .
$$

Yao \& Stewart (1995) give an almost identical expression for $U_{c}$, derived from large activation energy asymptotics. It is worth noting that the high sensitivity of the induction time $\tau$ to the post-shock temperature $T_{s}$ and hence shock velocity $U$ means the critical energy predictions of the CDR model will be very sensitive to the choice of $U_{*}$. Our choice here is by no means the definitive one, and this represents an area for future study.

\section{Comparison with experiment}

The global initiation criterion for spherically symmetric detonations, (6.4), is compared with various sets of experimental data in figures 19 to 21 . In all cases the initial conditions were approximately 1 bar and $300 \mathrm{~K}$. The values of $U_{*}, \tau_{*}, \gamma, Q$ and $\theta_{*}$ were determined as outlined in $\S 6$. The hydrocarbon reaction mechanism of Miller \& Bowman (1989) was used in the hydrogen and ethylene calculations for figures 19 and 20. A natural gas reaction mechanism from the Gas Research Institute (Bowman et al. 1995) was used in the calculations for figure 21, as this is a more recent mechanism which has been extensively tested for methane and ethane. In figures 19 and 20, the critical energy is plotted against equivalence ratio $\phi$. The ordinate $\beta$ in figure $21(a)$ is the volume ratio of $\mathrm{N}_{2}$ to $\mathrm{O}_{2}$, where 3.76 corresponds to air. 

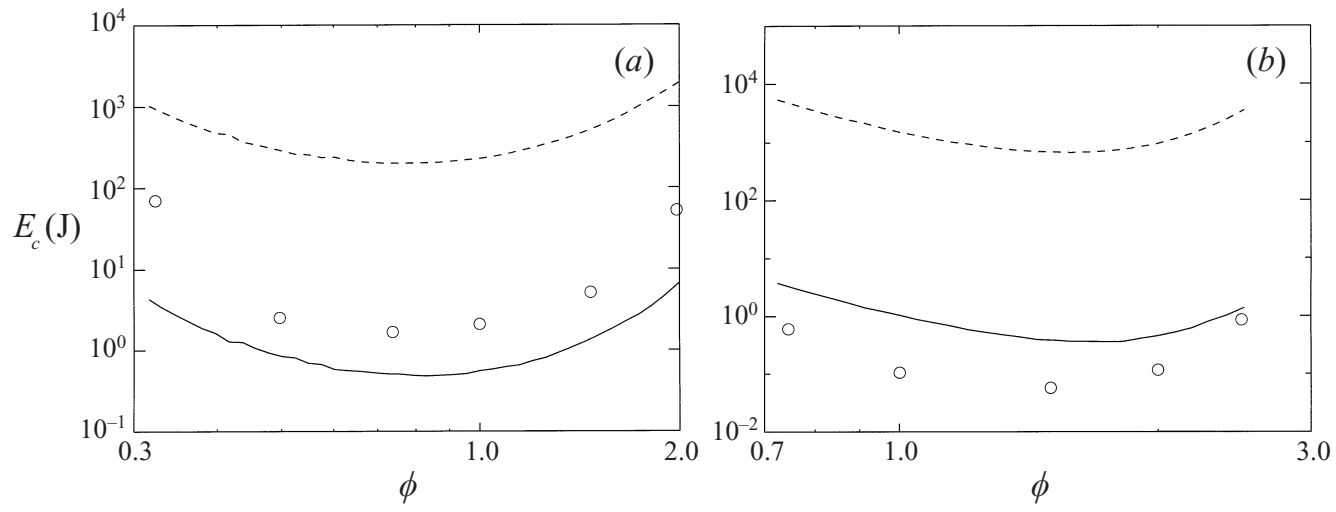

Figure 20. As figure 19 but $\circ$, experiment (Matsui \& Lee 1979). (a) $\mathrm{H}_{2}-\mathrm{O}_{2}$; (b) $\mathrm{C}_{2} \mathrm{H}_{4}-\mathrm{O}_{2}$.
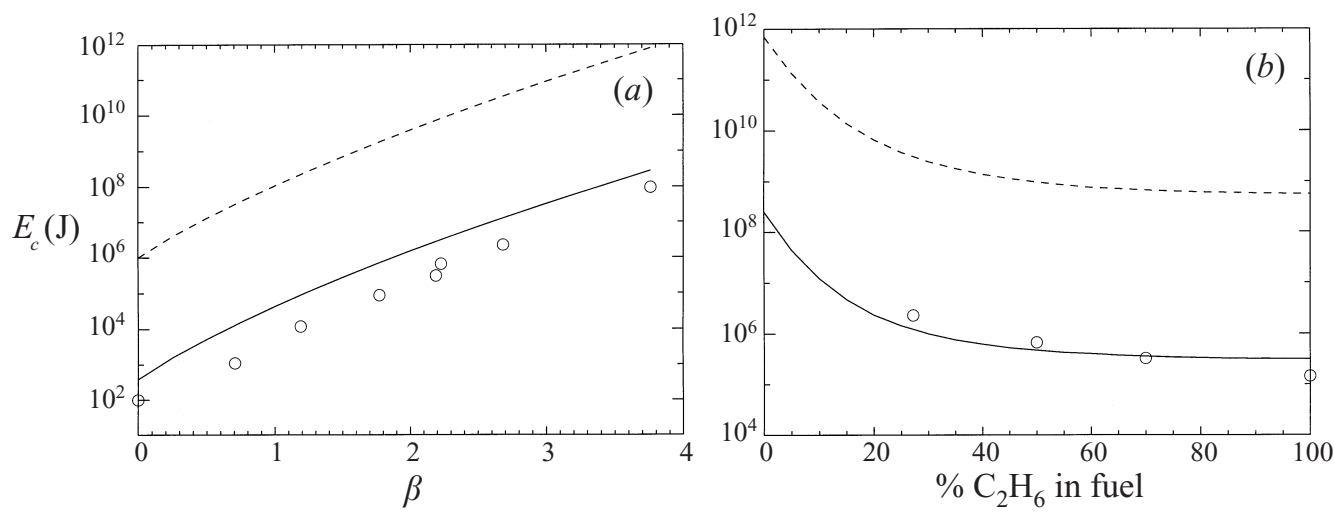

FiguRE 21. Comparison between theory and experiment for critical energy: — rate model, (6.4); - - , critical curvature model (He \& Clavin 1994), (7.3); o, experiment. (a) Stoichiometric $\mathrm{CH}_{4}-\mathrm{O}_{2}-\mathrm{N}_{2}$ (experimental data from Bull et al. 1976); (b) stoichiometric $\mathrm{CH}_{4}-\mathrm{C}_{2} \mathrm{H}_{6}$-air (experimental data from Bull, Elsworth \& Hooper 1979).

For comparison, the critical energy predictions of the critical curvature model (He \& Clavin 1994) are also shown in these figures. This model gives the critical energy as

$$
E_{c}=A_{j}\left(\frac{j+3}{2}\right)^{2} \rho_{0} U_{c}^{2} R_{c}^{j+1}
$$

where $R_{c}$ is the critical radius and $U_{c}$ is the corresponding shock velocity. Using the authors' asymptotic square-wave detonation model, the critical radius is given by

$$
R_{c}=\frac{8 \mathrm{e} j \theta_{C J}}{1-\gamma^{-2}} \Delta_{C J}
$$

where $\Delta_{C J}$ is the induction length for a $\mathrm{CJ}$ detonation. $U_{c}$ was given in (6.6). Then (7.1) becomes

$$
E_{c}=A_{j}\left(\frac{j+3}{2}\right)^{2}\left(\frac{8 \mathrm{e} j \theta_{C J}}{1-\gamma^{-2}}\right)^{j+1}\left(1-\frac{1}{2 \theta_{C J}}\right)^{2} \rho_{0} U_{C J}^{2} \Delta_{C J}^{j+1} .
$$


For spherically symmetric flow $(j=2)$,

$$
E_{c}=5.14 \times 10^{5} A_{2}\left(\frac{\theta_{C J}}{1-\gamma^{-2}}\right)^{3}\left(1-\frac{1}{2 \theta_{C J}}\right)^{2} \rho_{0} U_{C J}^{2} \Delta_{C J}^{3},
$$

and this equation was used to generate the curves in figures 19 to 21 .

The figures show that the critical decay rate (CDR) model generally gives critical energies about three orders of magnitude less than the critical curvature model. The CDR model also agrees with the experimental data to within an order of magnitude, except in the case of near-stoichiometric hydrogen-air. The agreement is particularly good in the hydrocarbon figures. The model slightly overpredicts the critical energy in most of these cases, but this is a substantial improvement on the large overprediction of the critical curvature model. Admittedly, the critical curvature model could be applied more accurately by computing the slightly curved quasi-steady $U-R$ solution using a real reaction mechanism and locating the critical point $U_{c}-R_{c}$, as described in He (1996). This would then be substituted into (7.1) rather than the approximate results of the square-wave model. However, use of the square-wave model to find the critical point is only slightly different and the critical energy predictions would be similar. Comparison between the CDR and critical curvature models is less conclusive in the hydrogen figures where the experimental data generally lie between the two models.

While discussing the critical curvature model of $\mathrm{He} \&$ Clavin (1994), it is instructive to compute the critical radius $R_{c}$ for the two computational cases presented in this paper, and compare with the computational results in figures 3 and 10. Assuming $\Delta_{C J} \approx \Delta_{1 / 2}$, then $\tilde{R}_{c} \approx R_{c} / \Delta_{C J}$, which can be computed directly from (7.2). For case A, $\tilde{R}_{c}=861$, which is much greater than the shock radius in the critical regime from the numerical simulations, $\tilde{R} \approx 225$. For case $\mathrm{B}, \tilde{R}_{c}=492$, which is also considerably greater than the shock radius in the critical regime for the first critical energy, $\tilde{R} \approx 285$. This supports our assertion that the critical radius is not the controlling variable for direct initiation.

The experimental U-shaped curve for hydrogen-air in figure $19(a)$ is a significantly different shape from that of the models. This indicates a more complicated behaviour for this mixture than is accounted for in the models. Near-stoichiometric hydrogenair mixtures have an unusually long recombination zone relative to the size of the induction zone (Shepherd 1986), but this property does not exist away from stoichiometry. Models based purely on analysis of the induction zone will not include the effect of the recombination zone. This may explain the CDR model's large discrepancy with the experimental data for near-stoichiometric hydrogen-air.

The slight deviation of the experimental data from the CDR model for rich ethylene-air mixtures is due to the fact that the Miller-Bowman hydrocarbon mechanism does not include any large hydrocarbon molecules. Rich mixtures will involve the recombination of ethylene molecules early in the induction zone to form large hydrocarbons not included in the mechanism. Hence the mechanism is not expected to accurately compute the dynamic parameters in the rich regime.

While the agreement between experiment and the CDR model appears approximate at best, it must be noted that the error bars on both the experimental data and model predictions are quite large. The model relies on an accurate reaction mechanism for the computation of the induction time $\tau_{*}$ and global activation energy $\theta_{*}$. Despite extensive efforts in the development of these mechanisms in recent decades, there is still considerable uncertainty in their accuracy, particularly when applied to the 
high pressures associated with detonations in gases initially at standard pressure. Two seemingly satisfactory mechanisms for the same mixture often give induction times that differ by a factor of 2 or more. Since the dynamic parameters $\tau_{*}$ and $\theta_{*}$ are each cubed in the spherically symmetric model equation (6.4), this could give an order of magnitude error in the predicted critical energy. There are also a number of sources of uncertainty in the experimental data, as follows.

(a) The data sets presented in figures 19 to 21 consist mostly of averages of 'Go' and 'NoGo' experiments which bracket the critical energy quite coarsely.

(b) There are significant differences in experimental data obtained from various types of initiation sources. The most common sources are exploding wires, electrical sparks and solid explosives. It is often unclear exactly how much of the nominal source energy actually goes into the gas, and also whether the energy is deposited sufficiently rapidly and compactly to act like an instantaneous point source. These uncertainties are particularly significant for exploding wires and electrical sparks. For this reason, we have chosen not to use any experimental data from these two initiation sources. The data in figures 19 and 21 used high explosives as the initiation source.

(c) For very sensitive mixtures with $E_{c}$ of the order of $1 \mathrm{~J}$ or less, typical of nearstoichiometric fuel- $\mathrm{O}_{2}$ mixtures, use of even high explosives for the initiation source becomes difficult. The electrical charge used to initiate the small piece of high explosive is no longer a negligible energy source. Furthermore, it is unlikely that a clean spherical detonation can be formed in the high explosive before the blast wave travels into the gas. For this reason, no satisfactory experiments have been performed to date with fuel- $\mathrm{O}_{2}$ mixtures using a high-explosive initiation source. Hence, we have chosen not to consider any fuel- $\mathrm{O}_{2}$ critical energy data determined from point initiation experiments. The data in figure 20 have been taken from Matsui \& Lee (1979) who actually performed critical tube diameter experiments with planar detonations and converted the data to critical energies using a phenomenological model known as the work done model (Lee \& Matsui 1977). These data are thus subject to errors introduced by the use of the model, which is at best order-of-magnitude accurate (Benedick et al. 1986).

(d) It is difficult to perform direct initiation experiments with insensitive mixtures that have large critical energies. A very large experimental facility is required if the velocity or pressure profiles are to be conclusive as to whether a detonation is successfully initiated before wave reflection occurs. The initiator energy must be small compared to the total energy inside the experiment containment. In the past, several experiments with rich or lean fuel-air mixtures have suffered from considerable uncertainty due to this factor. The same can also be said of near-stoichiometric fuel-air mixtures with very insensitive fuels such as methane.

The above arguments demonstrate the large uncertainties in most experimental data on critical energies. This is particularly true of fuel-oxygen mixtures, for which various experimental results often differ by orders of magnitude. An example is hydrogen, where the experiments of Litchfield, Hay \& Forshey (1963) with $\mathrm{H}_{2}-\mathrm{O}_{2}$ mixtures using exploding wire and electrical spark initiation sources gave critical energies respectively nearly one and two orders of magnitude higher than those of Matsui \& Lee (1979) shown in figure 20.

\section{Conclusions}

The one-dimensional reaction-zone structure in gaseous detonations is a competition between heat release, wave curvature and unsteadiness. In direct initiation by 
a blast wave, numerical simulations with a simple one-step reaction model and Arrhenius reaction rate have demonstrated that the dominant balance is between heat release and unsteadiness. Hence the primary physical mechanism by which a detonation may fail to initiate is excessive unsteadiness in the reaction zone arising from the deceleration of the leading shock. The critical amount of unsteadiness was determined from a large activation energy asymptotic analysis of the reactive Euler equations with the one-step reaction model. The local initiation model was validated through quasi-unsteady calculations with real gas kinetics. It was found that the model agreed with the numerical calculations to within $40 \%$, for a number of fuel-oxidizer mixtures over a wide range of stoichiometries.

An analytical equation for the critical energy was developed from the local initiation model by means of an assumed blast wave velocity profile. Closure can be obtained by applying the local initiation model at a prescribed critical point in the velocity profile. The optimal choice of this point remains an unresolved issue, and in this paper we have made an ad-hoc choice to use the shock velocity corresponding to the critical radius in the quasi-steady slightly-curved nonlinear detonation relationship. The analytical equation thus obtained was found to give order of magnitude agreement with numerical and experimental data. The agreement with experiment is quite satisfactory at present. With large uncertainties in both experimental data and theoretical reaction mechanisms, we cannot hope to validate direct initiation models against experiment to more than an order of magnitude comparison. Improved accuracy in experiments and reaction mechanisms is required before more detailed validation will be possible.

We propose the CDR model as a model for spherical, cylindrical and planar direct initiation. However, we have only validated it with numerical and experimental data in the case of spherical detonations. The application to cylindrical and planar detonations is speculative and the possible subject of further research. Validation of the planar case with numerical simulations using the one-step Arrhenius reaction model may be complicated by the difficulty of distinguishing 'Go' and 'NoGo' initiation events. This complication was observed by Mazaheri (1997). It is an effect of the slow rate of blast wave decay in the planar case, coupled with the one-step model's non-physical properties at low temperatures and the inevitable completion of reaction at long but finite times. However, this is a numerical artifact of the one-step model and not something observed in real detonations, so we do not believe it is a reason for discounting any direct initiation model in the planar case.

The numerical simulations presented here for case B also identified an interesting phenomenon, that of a non-unique critical energy. Whether this is a physical phenomenon or another artifact of the one-step model is a question for further study. Simulations with realistic thermochemistry would be very illuminating in this regard. With this non-uniqueness in mind, we chose to examine only the lower critical energy since there appeared to be some universality of behaviour between cases A and B. In that case, there was a clear and sudden distinction between marginally subcritical and marginally supercritical initiation cases that occurred in the initial blast wave decay. Our model is an attempt to explain this behaviour. Clearly, our global initiation model cannot hope to explain the more complicated dynamics associated with the higher critical energy, since it is based on an assumed monotonically decaying blast wave law. So at best, the model gives a lower bound estimate for the critical energy.

The authors would like to thank Dr Tom Jackson for the provision of the onedimensional detonation stability code used to produce figure 1 . This research has been 
supported by Los Alamos National Laboratory-subcontract 319AP0016-3L under DOE Contract W-7405-ENG-36.

\section{Appendix}

In $\S 3.2$, the curvature term in the temperature-reaction-zone structure equation (2.13) was seen to be much smaller than the unsteadiness term, numerically. Here we examine the ratio of these terms analytically. Since the numerics suggested each of these terms was constant in the induction zone, prior to failure, we can approximate their ratio by their initial ratio just behind the leading shock. Using (2.13), and the strong-shock perfect-gas jump conditions (4.6), the ratio of curvature to unsteadiness is

$$
\frac{j}{3} \frac{\gamma-1}{\gamma+1} \frac{U^{2}}{R} \frac{1}{\mathrm{~d} U / \mathrm{d} t}
$$

To compute this ratio, a shock velocity profile is required. As detailed in $\S 6$, we adopt the modified Taylor-Sedov solution for a strong point blast with chemical energy release. Using (6.1a) and (6.2), the absolute value of the above ratio reduces to

$$
\frac{2}{3} \frac{j}{j+1} \frac{\gamma-1}{\gamma+1}\left(1+\frac{j+2}{j+1} \frac{B_{j} Q}{U^{2}}\right) \exp \left\{-\frac{B_{j} Q}{(j+1) U^{2}}\right\} \text {. }
$$

This expression appears quite complicated, but if we evaluate it in the spherical case $(j=2)$ at the failure point $U=U_{*}$ for the real gas mixtures studied in figures 19 to 21 , we find it is almost constant at 0.1 , with the maximum value for any of the mixtures or stoichiometries being only 0.12 . In the cylindrical case $(j=1)$, the ratio will be even less. Hence, from an analytical consideration of the terms in the temperature-reaction-zone structure equation, we conclude that the curvature term is at least one order of magnitude smaller than the unsteadiness term in the critical region of the flow. Thus it is justifiably omitted from the analysis.

\section{REFERENCES}

Benedick, W. B., Guirao, C. M., Knystautas, R. \& Lee, J. H. S. 1986 Critical charge for the direct initiation of detonation in gaseous fuel-air mixtures. Prog. Astronaut. Aeronaut. 106, 181-202.

Bowman, C. T., Hanson, R. K., Davidson, D. F., Gardiner, JR., W. C., Lissianski, V., Smith, G. P., Golden, D. M., Frenklach, M. \& GoldenberG, M. 1995 GRI-Mech 2.11. http://www.me.berkeley.edu/gri_mech/.

Bull, D. C., Elsworth, J. E. \& Hooper, G. 1979 Susceptibility of methane-ethane mixtures to gaseous detonation in air. Combust. Flame 34, 327-330.

Bull, D. C., Elsworth, J. E., Hooper, G. \& Quinn, C. P. 1976 A study of spherical detonation in mixtures of methane and oxygen diluted by nitrogen. J. Phys. D: Appl. Phys. 9, 1991-2000.

Clarke, J. F., Kassoy, D. R., Meharzi, N. E., Riley, N. E. \& Vasantha, R. 1990 On the evolution of plane detonations. Proc. R. Soc. Lond. A 429, 259-283.

Clarke, J. F., Kassoy, D. R. \& Riley, N. 1986 On the direct initiation of a plane detonation wave. Proc. R. Soc. Lond. A 408, 129-148.

Fickett, W. \& Davis, W. C. 1979 Detonation. University of California Press.

Frank-KamenetskiI, D. A. 1969 Diffusion and Heat Transfer in Chemical Kinetics. Plenum.

Glaister, P. 1988 An approximate linearised Riemann solver for the Euler equations for real gases. J. Comput. Phys. 74, 382-408.

HE, L. 1996 Theoretical determination of the critical conditions for the direct initiation of detonations in hydrogen-oxygen mixtures. Combust. Flame 104, 401-418.

He, L. \& Clavin, P. 1994 On the direct initiation of gaseous detonations by an energy source. J. Fluid Mech. 277, 227-248. 
Kee, R. J., Rupley, F. M. \& Miller, J. A. 1989 CHEMKIN-II: A Fortran chemical kinetics package for the analysis of gas-phase chemical kinetics. Tech. Rep. SAND89-8009. Sandia National Laboratories.

Korobeinikov, V. P. 1968 Point explosion in a detonating gas. Sov. Phys.-Dokl. 12, 1003-1005.

Korobeinikov, V. P. 1991 Problems of Point-Blast Theory. American Institute of Physics.

LeE, H. I. \& Stewart, D. S. 1990 Calculation of linear detonation instability: One-dimensional instability of plane detonation. J. Fluid Mech. 216, 103-132.

LeE, J. H. S. 1977 Initiation of gaseous detonation. Ann. Rev. Phys. Chem. 28, 75-104.

LeE, J. H. S. 1984 Dynamic parameters of gaseous detonations. Ann. Rev. Fluid Mech. 16, 311-336.

LeE, J. H. S. \& Higgins, A. J. 1999 Comments on criteria for direct initiation of detonation. Phil. Trans. R. Soc. Lond. A 357, 3503-3521.

Lee, J. H. S. \& Matsui, H. 1977 A comparison of the critical energies for direct initiation of spherical detonations in acetylene-oxygen mixtures. Combust. Flame 28, 61-66.

Litchfield, E. L., Hay, M. H. \& Forshey, D. R. 1963 Direct electrical initiation of freely expanding gaseous detonation waves. In 9th Symp. (Intl) on Combustion (ed. W. G. Berl), pp. 282-286. Academic.

Matsui, H. \& LeE, J. H. S. 1979 On the measure of the relative detonation hazards of gaseous fuel-oxygen and air mixtures. In 17th Symp. (Intl) on Comubstion (ed. G. M. Faeth), pp. 1269-1280. The Combustion Institute.

MAZAHERI, K. 1997 Mechanism of the onset of detonation in blast initiation. PhD thesis, Department of Mechanical Engineering, McGill University.

Miller, J. A. \& Bowman, C. T. 1989 Mechanism and modeling of nitrogen chemistry in combustion. Prog. Energy Combust. Sci. 15, 287-338.

QUiRk, J. J. 1998 (i) Amrita: A computational facility (for CFD modelling), (ii) Amr_sol: Design principles and practice. In 29th Computational Fluid Dynamics lecture series (ed. H. Deconinck). Von Karman Institute, http://www.amrita-cfd.org/doc.

REYNOLDS, W. C. 1986 The element potential method for chemical equilibrium analysis: Implementation in the interactive program STANJAN. Tech. Rep. Stanford University, Dept of Mech. Engng.

RoE, P. L. 1986 Characteristic-based schemes for the Euler equations. Ann. Rev. Fluid Mech. 18, $337-365$

Sedov, L. I. 1959 Similarity and Dimensional Methods in Mechanics, 4th edn. Academic.

Shampine, L. F. \& WATts, H. A. 1970 ZEROIN, a root-solving code. Tech. Rep. SAND SCTM-70631. Sandia National Laboratories.

Shampine, L. F. \& WatTs, H. A. 1979 DEPAC - Design of a user oriented package of ODE solvers. Tech. Rep. SAND79-2374. Sandia National Laboratories.

Shepherd, J. E. 1986 Chemical kinetics of hydrogen-air-diluent detonations. Prog. Astronaut. Aeronaut. 106, 263-293.

Stewart, D. S., Aslam, T. D. \& Yao, J. 1996 On the evolution of cellular detonation. In 26th Symp. (Intl) on Combustion (ed. A. R. Burgess \& F. L. Dryer), pp. 2981-2989. The Combustion Institute.

Stewart, D. S. \& Bdzil, J. B. 1988 The shock dynamics of stable multi-dimensional detonation. Combust. Flame 72, 311-323.

TAYLOR, G. I. 1950 The formation of a blast wave by a very intense explosion. I. Theoretical discussion. Proc. R. Soc. Lond. A 201, 159-174.

YAO, J. \& StewaRT, D. S. 1995 On the normal shock velocity-curvature relationship for materials with large activation energy. Combust. Flame 100, 519-528.

YaO, J. \& Stewart, D. S. 1996 On the dynamics of multi-dimensional detonation. J. Fluid Mech. 309, 225-275.

Zel'dovich, I. B., Kogarko, S. M. \& Simonov, N. N. 1956 An experimental investigation of spherical detonation of gases. Sov. Phys. Tech. Phys. 1 (8), 1689-1713. 